\title{
Development of a national medical leadership competency framework: the Dutch approach
}

Wouter A. Keijser ${ }^{1,2^{*}}$ D , Henricus J. M. Handgraaf ${ }^{3}$, Liz M. Isfordink ${ }^{4}$, Vincent T. Janmaat ${ }^{5}$, Pieter-Paul A. Vergroesen ${ }^{6}$, Julia M. J. S. Verkade ${ }^{7}$, Sietse Wieringa ${ }^{8,9}$ and Celeste P. M. Wilderom ${ }^{1}$

\begin{abstract}
Background: The concept of medical leadership (ML) can enhance physicians' inclusion in efforts for higher quality healthcare. Despite ML's spiking popularity, only a few countries have built a national taxonomy to facilitate ML competency education and training. In this paper we discuss the development of the Dutch ML competency framework with two objectives: to account for the framework's making and to complement to known approaches of developing such frameworks.
\end{abstract}

Methods: We designed a research approach and analyzed data from multiple sources based on Grounded Theory. Facilitated by the Royal Dutch Medical Association, a group of 14 volunteer researchers met over a period of 2.5 years to perform: 1) literature review; 2) individual interviews; 3) focus groups; 4) online surveys; 5) international framework comparison; and 6) comprehensive data synthesis.

Results: The developmental processes that led to the framework provided a taxonomic depiction of ML in Dutch perspective. It can be seen as a canonical 'knowledge artefact' created by a community of practice and comprises of a contemporary definition of ML and 12 domains, each entailing four distinct ML competencies.

Conclusions: This paper demonstrates how a new language for ML can be created in a healthcare system. The success of our approach to capture insights, expectations and demands relating leadership by Dutch physicians depended on close involvement of the Dutch national medical associations and a nationally active community of practice; voluntary work of diverse researchers and medical practitioners and an appropriate research design that used multiple methods and strategies to circumvent reverberation of established opinions and conventionalisms.

Implications: The experiences reported here may provide inspiration and guidance for those anticipating similar work in other countries to develop a tailored approach to create a ML framework.

Keywords: Medical leadership, National competency framework, Medical education, Qualitative, Design research

\section{Background}

\section{Emergence and discoursehes}

Over the past decade the concept of medical leadership (ML) has emerged as a result of various contestations over physicians' changing roles and impact on healthcare delivery [1]. Supposedly, ML emerged during attempts to include

\footnotetext{
* Correspondence: wouter@keijser.com

${ }^{1}$ Faculty of Behavioural, Management and Social Sciences (BMS) Change,

Management and Organizational Behavior (CMOB), University Twente, Enschede, The Netherlands

2DIRMI Foundation, Utrecht, The Netherlands

Full list of author information is available at the end of the article
}

more medical professionals in quality and safety improvements and healthcare transformation [2, 3]. In recent years, ML has been increasingly theorized as being a part of physicians' attempts to re-professionalize $[4,5]$.

The discourse of ML can be explained in two ways. First, it can be conceived as a revision of physicians' professional identity as a response to institutional disruptions, which increasingly affect physicians' traditional dominant and autonomic positions [6-8]. Secondly, rapid changes in daily healthcare practices warrant ML efforts $[9,10]$. The changing role of physicians is influenced by various factors, including:

(c) The Author(s). 2019 Open Access This article is distributed under the terms of the Creative Commons Attribution 4.0 International License (http://creativecommons.org/licenses/by/4.0/), which permits unrestricted use, distribution, and reproduction in any medium, provided you give appropriate credit to the original author(s) and the source, provide a link to the Creative Commons license, and indicate if changes were made. The Creative Commons Public Domain Dedication waiver (http://creativecommons.org/publicdomain/zero/1.0/) applies to the data made available in this article, unless otherwise stated. 
technological innovations; patient empowerment; system reforms; and rising economic constraints. Over the years, such developments have ignited the need for agency to rebalance the shifting interprofessional arrangements between physicians and other field actors. Physicians' skill sets have been in transit within these processes, from individualistic clinical experts or "heroic lone healers" ([11]: p57) to collaborative leaders in change and improvement [12].

A transition to a more collective approach to practicing medicine is well represented in the current literature on ML (Fig. 1). Moreover, the literature provides indications for the beneficial effects of ML e.g., on clinical and organizational outcomes [13], as well as on physician's burnout reduction [14]. Yet, enhancement of rigor in research on ML is wanted [15].

Internationally, the physician's role of 'leader' was recently formalized through replacement of the former 'manager' role in the CanMEDS framework [16]. Also, various forms of ML training are increasingly being offered, including the appearance of ML competency programs in formal curricula [17]. Triggered by a variety of precipitating activities and an increasing appetite for ML within as well as outside national medical communities $[1,18]$, interestingly, in several countries a comprehensive national ML competency framework has been developed and implemented $[1,3,19,20]$. As it appears, ML is here to stay. ML is following a national discourse in which the creation of a national taxonomy on ML is an essential component $[1,3,5,13,18-20]$. To our knowledge, to date, there has been no publication providing detailed insights on 'the making of' such an artifact. This paper provides an account of the development of a national ML competency framework, exemplified by the approach taken in the Netherlands.

\section{Medical leadership competency frameworks}

Specifying professional behavior and performance, competencies form part of the shared identity of a profession and its members. Formally defining them can contribute to explicating a profession's objectives to others [21]. ML frameworks (that comprise the relevant competencies of a physician's role(s) in leading [16]), have been subject to disputes [22, 23]. Generally, leadership frameworks can benefit from a collective understanding of leadership practices and educational content [20]. Detailing desirable behaviors, such frameworks, or 'knowledge artefacts', help convey clear meaning, and align classifications of terms, concepts, and elements [24]. Furthermore, competency frameworks "constitute a blueprint for optimal performance" which individuals are expected to master them ([25]: p.870). Such frameworks also answer the need to establish consistent standards of practices across settings, including evaluating outcomes of competency development [26]. Furthermore, competency frameworks can provide practitioners, educators and human resource professionals with an outline to appropriately choose or develop educational activities and assessments to enhance proficiency $[25,27]$. Without a common and welldesigned vocabulary on the concept of ML, applicable in daily practice and in education, any effective enactment of it by physicians, educators, managers, policy-makers and others

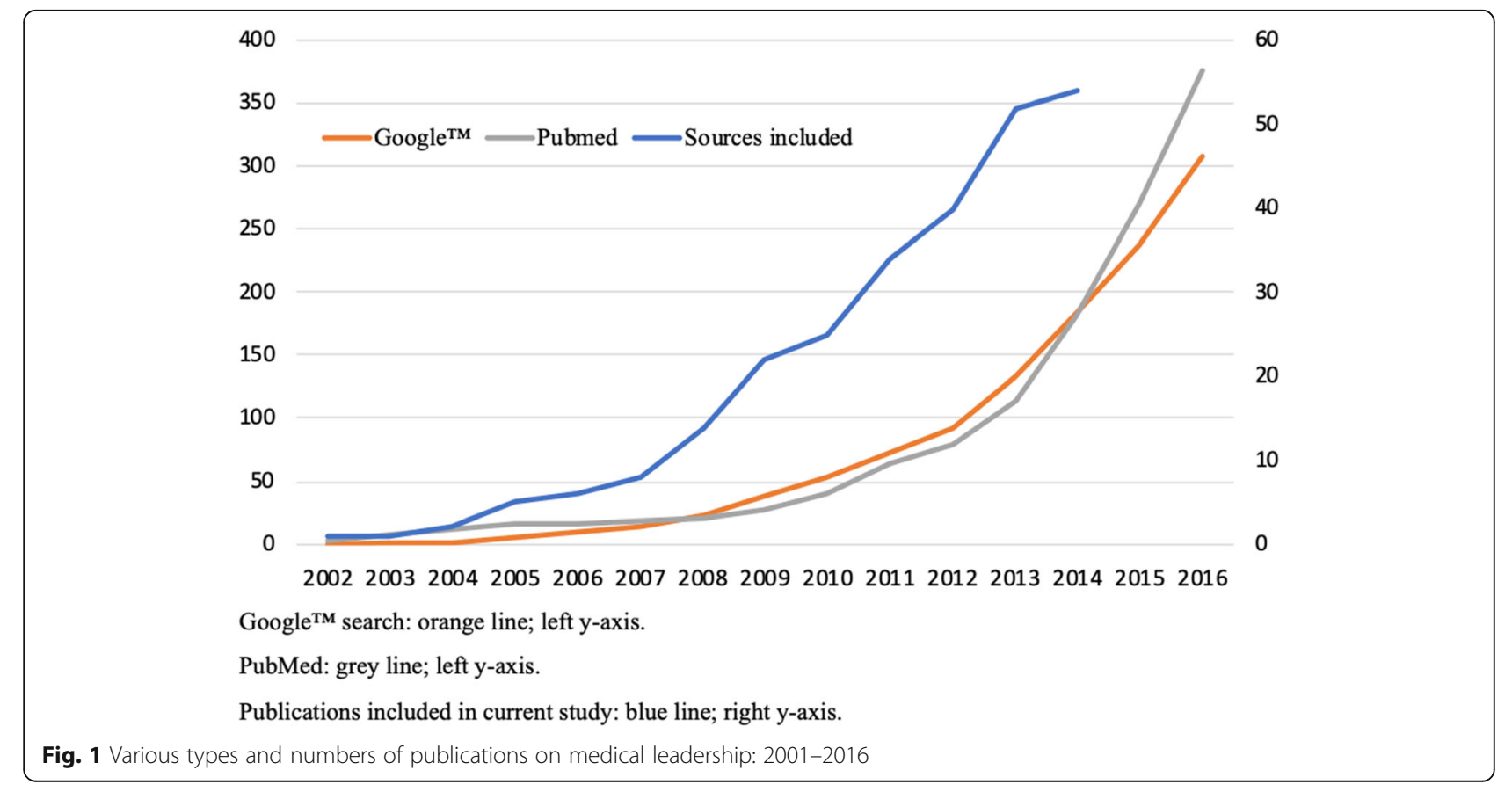


might remain ambiguous, consequently hampering effective improvements and transformation in healthcare [18-20].

Thus, without adequate explanations for the meaning of the competencies required by the relatively new and 'trendy' ML concept, enshrined within the notion of 'physicians as leaders', could trigger (Babylonian) misconceptions. It could, for example, kindle interprofessional boundary battles when physicians enacting ML are (mis) perceived as 'being the boss': possibly reinforcing healthcare's notorious hierarchical culture of professional power. Also, misunderstandings can arise from unclear distinctions between $\mathrm{ML}$ and other function-related forms of leadership e.g., 'clinical leadership' (implying all healthcare professionals), or 'managerial' ML (indicating physicians in hybrid leadership roles) [28]. Competency frameworks can help raise awareness of the meaning of leadership, by bringing a lexicon with which individuals, organizations, educators and others can further debate on the nature of physician leadership, and its associated value to organizations, professions and ultimately to patients [29]. Also, a precise definition of ML, as sought after in this Dutch project, could help mitigate such misapprehensions.

\section{Framework development}

For various reasons, the construction of a national framework, suitable to function during times of unprecedented institutional change in a healthcare arena, can be a challenging task [5]. Firstly, although extant ML frameworks have proven their value in various countries, no generic process map for their development has been published to date. Secondly, defining professional competencies is often based on the existing generation of professionals' views and experiences, despite consultations of large groups of peers who are invited to score concepts of new 'best practices' that are predefined by those elites. Such an approach risks a continuous reinforcement of "the current thinking of a limited few who occupy dominant professional positions" ([30]: p. 452) within the medical community or the politics surrounding it. But professional competency frameworks are expected to be societally responsive [21]. Any new medical framework must thus function as a timely and appropriate illumination of patient care as well as societal needs and demands vis-à-vis physicians [10]. Thirdly, independence and efficiency are required from those who construct the medical frameworks. Moreover, 'policy community' type of project organizations (that comprise organizing various streams of discussion groups in and between professional, healthcare governance and other bodies and associations towards a series of consensus meetings etc.) has been noted to slow down innovation. Also, a politically tainted 'governing of the souls' (e.g., solely centrally organized, top-down approaches of designing new policy and practice) can influence physicians' subjectivism in re-professionalization processes $[4,5]$. A fourth difficulty that can be encountered pertains to the roles of regulatory agencies and professional associations in deploying new frameworks. Involvement of these stakeholders can be crucial for the sustainability of any framework implementation [31] because they can delay new medical realities, due to competing priorities resulting from their relations with entrenched constituents. Finally, a competency framework is not static; it needs to be chaperoned over time to retain its accuracy and for it to remain contemporary [21].

\section{The study objectives}

In the absence of detailed publications explaining the development of a national ML competency framework, this paper's main objective is to provide a design-process description of the Dutch case study, to inspire or guide others contemplating to undertake similar work in other countries [32]. In particular, our community of practice approach might add to possible avenues of creating these national artifacts. Below we explain in detail the methodological foundation on which version 1.0 of the Dutch Medical Leadership (DML) framework was constructed. The final version of the process depicted below can be found here: https://osf.io/qknds/.

\section{Methods \& design process}

The following design research methods were used: systematic literature review; individual interviews; Groundedtheory type data analysis and synthesis; comparison of the framework's initial 0.1 version with other national ML frameworks; validation of the 0.2 DML framework version through focus groups (FG) and an online survey; and translation of the Dutch version into English (see, Fig. 2).

\section{Setting and actors}

Given the absence of a Dutch ML taxonomy and anticipating an increase in the use of unofficial translations of foreign (and especially UK) frameworks, this project was initiated in early 2013 by researchers from the University of Twente (UT) and members of the Platform Medical Leadership (PML). PML ${ }^{1}$ is a non-profit Dutch foundation based on the (free of charge) membership of approximately 200 Dutch individuals (2014), primarily physicians. Since its establishment in 2012, PML in team with the research group has been functioning as a 'Community of Practice' (CoP) in ML: a group of people "who share a concern, a set of problems or a passion about [ML] and who deepen their knowledge and expertise in this area by interacting on an ongoing basis ... [and] ... create tools, standards, generic designs, manuals, and other 


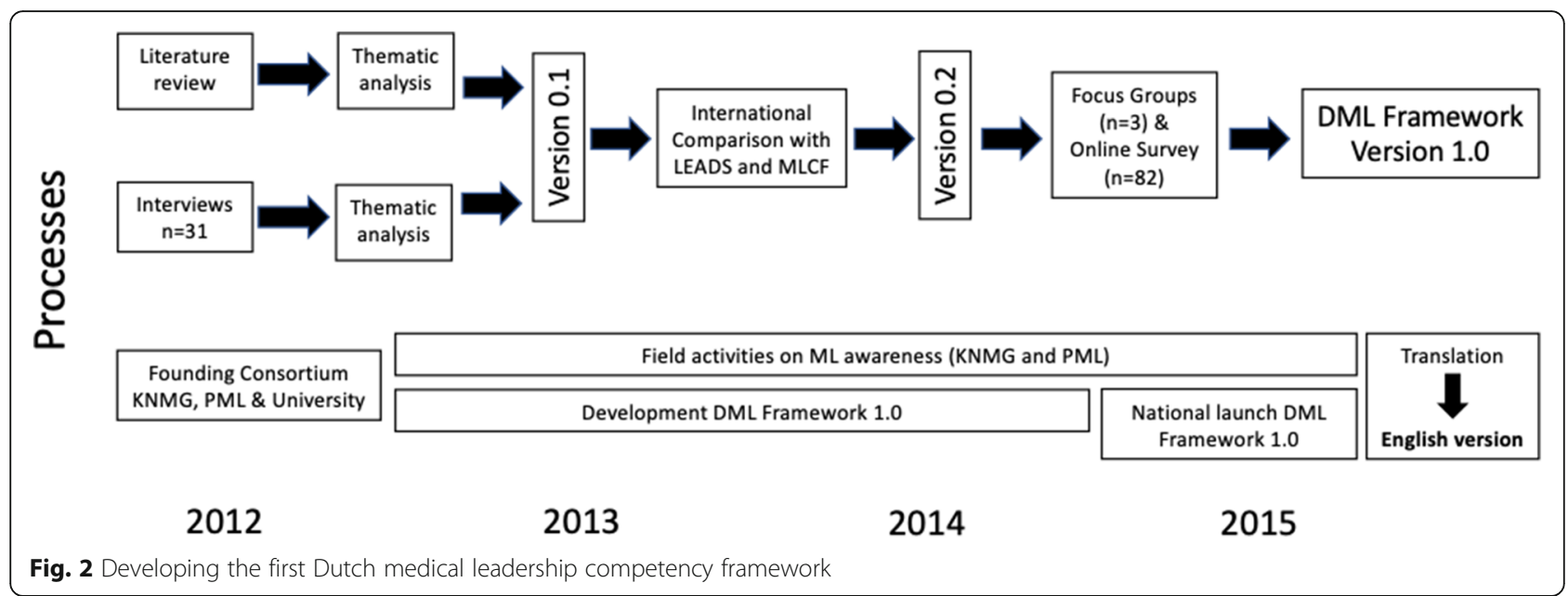

documents" ([33]: 4-5). A national consortium consisting of PML, UT and the Royal Dutch Medical Association (KNMG) began, with the objective to create and launch a national, evidence-based, open-access ML framework. PML and UT researchers agreed to engage in the collective long-term dual custodianship of the envisioned framework's development and ongoing maintenance. After its development, under the academic scrutiny of the UT, and under the auspices of PML and KNMG, this consortium launched the 1.0 DML framework version in December 2015 (see: https://osf.io/qknds/) [18].

Based on the limited literature about resources and approaches used in the development of other frameworks as well as on input from international experts ${ }^{2}[3,19,20]$, we contend that the way the DML framework was constructed differs in that it used an independent community or practice approach [33]. Before describing the methodologies we applied, we first want to discuss the strategic rationale for this approach and the context in which the work was done.

\section{Research group}

The framework's research group of 14 individuals had an active core of eight persons, including: six physicians (with backgrounds in: primary care (2); surgery (2); internal medicine (1); and change management and coaching (1)); one MSc-level registered nurse / MSc health scientist; and a full-tenured professor in organizational behavior and leadership studies. The additional six individuals were: a KNMG policy advisor; a medicaleducation expert; a statistician; and three UT student assistants. Except for the two topic experts (WK; CW), the core group members were mainly recruited from the

\footnotetext{
${ }^{1}$ PML's mission: "A cultural shift is warranted in which physicians, from the start of medical training, are stimulated to and educated in engaging adequately in medical organizations".
}

PML network. Others were invited based on interest, pragmatism and required expertise. Twelve of the 14 participated on a voluntary basis; the other two were remunerated (i.e., the university statistician and the KNMG policy advisor). The composition of the core group did not alter throughout the framework's developmental process. Members of both groups engaged in specific tasks, in subgroups of varying sizes (Table 1); one core group member had a central coordinating role (WK). All eight researchers were involved in final consensus forming and prime decision making throughout all the phases.

Over a period of 2.5 years, the researchers convened during 34 sessions, mostly face-to-face, at central locations in the Netherlands (at the KNMG premises) or via teleconference $\left(\mathrm{Skype}^{\mathrm{m} x}\right)$. These sessions involved either the entire core group or subgroups with various compositions of the entire group of researchers, lasting typically between approximately 1.5 to $5 \mathrm{~h}$ (Table 1 ). During this period, consortium representatives convened on 5 occasions: to discuss the project's progress, relevant field activities, preparation for the framework's launch and for other specific issues such as, for example, to make a taxonomical distinction between medical management, medical leadership and clinical leadership; the pace of the developmental process; and to share relevant 'soundings' from the field.

\section{Modus operandi}

The researchers ensured an enactment of high-quality activities by building on prior experiences and expert advice $^{3}$ During three preparatory sessions, the researchers'

\footnotetext{
${ }^{2}$ The authors were aware of approaches taken in the creation of ML frameworks by means of interviews (part of a preceding publication [1]) and personal communications with ML experts in the United Kingdom, Canada, Australia, New Zealand and Denmark, all being involved in national ML activities in these countries (see also: ${ }^{(2)}$ ).
} 
Table 1 Researchers' work sessions and subgroup sizes ${ }^{\mathrm{a}}$

\begin{tabular}{ll}
\hline & Number of sessions \\
\hline Core group work (In total: 8 people) & 3 \\
1. Research methodology \& preparations & 3 \\
2. Literature review analysis & 2 \\
3. Interviews' analysis & 2 \\
4. Synthesis and editing & \\
Subgroups (In total: 14 persons) & 4 \\
a. Literature review (6 persons) & 6 \\
b. Interviews and focus groups (6 persons) & 2 \\
c. International comparison (3 persons) & 5 \\
d. Version editing (4 persons) & 3 \\
e. Definition (3 persons) & 3 \\
f. Translation (4 persons) & 34 \\
TOTAL &
\end{tabular}

${ }^{a}$ Core group members also participated in subgroups

set of modus operandi was enshrined in four principles that were executed throughout the cycle of framework making, encompassing:

\section{Autonomy}

Responsibility for scientific rigor and quality of the framework's design: the researchers operated according to academic autonomy, parallel to the activities of the other consortium members (KNMG and PML) who were dedicated to deploying various activities (conferences; publications; workshops; etc.) to raise awareness among Dutch physicians of the topic before and after the framework's launch $[18,23]$.

\section{Neutrality}

The researchers operated under the academic guidance of the $\mathrm{UT}^{4}$ a university chosen for: (1) not harboring a medical school in order to guarantee independence and acceptability for all national medical universities by avoiding competition, (2) to reduce possible bias regarding the ML concept [5], and (3) having long-established international expertise in leadership research.

\section{Pluriform research group}

Most of the 14 researchers ${ }^{5}$ were practitioners with various clinical backgrounds. They had no prior experience in (medical) leadership research or practice; except for two experts [30, 34].

\footnotetext{
${ }^{3}$ Sources entailed: reports; publications and books on ML frameworks in UK, Canada, New Zealand and other sources as well as personal conversations with other scholars and international topic experts (expert names: on request).

${ }^{4}$ University of Twente's strategic mission intertwines the realms of 'high tech' and 'human touch', also in pursuit of effective transformation and improvement of healthcare systems.
}

\section{Topic expertise}

Two ML topic experts (WK and CW) led the development process, and also chaired most of the core and subgroup sessions. Neither participated in group voting procedures or consensus processes. Other authorities were asked for input where needed.

Although relatively small in size (in terms or financial resources as well as persons), the research group, which functioned according to the four principles, collaboration with other members of the multifaceted wider community of practice enabled a distinct balancing between inviting new ideas while nourishing existing 'ways of working'. The multiple sessions, with varying composition of people from various background, combined with numerous other ML related (national and local) activities and assemblies organized by the PML, KNMG and other groups (which were increasingly reported in professional and lay public media, during the period of the development [18]), importantly contributed to a collective and multileveled creation of the framework [35]. In fact, the development of the DML framework as described below, was couched in an intangible national 'knowledge interaction' [35]. Social science-oriented analyses of national ML discourses are being delivered by various scholars and contribute to an understanding of the dynamics of the emergence of new phenomena such as ML $[1,4,18]$. This paper's scope is the actual development process, to which we will turn to now.

\section{Methodological appropriateness and quality}

On disregarding the option to translate, adapt and validate existing foreign ML frameworks, we sought the highest possible (cultural) validity by constructing the Dutch ML framework from scratch [34]. In the absence of route maps for such a development [5], we first established a methodological approach and research plan. These were designed to ensure embedment of the framework's design in: (1) methodological rigor; (2) medical professionalism; and (3) future-proof societal relevance [21]. We set out to frame educational constructs and outcomes related to ML behavior which were applicable to Dutch physicians [23]. Therefore, we chose an unproblematized, realist approach providing a "direct window onto the world view" through various data sources and modes of synthetization ([36]: p5).

We collected data through 1) a literature review of scientific and grey literature; 2) field interviews; 3) focus groups of medical professionals; and 4) online surveys as discussed in detail below. We performed comprehensive data analysis and synthesis data which included comparison with international frameworks.

\footnotetext{
${ }^{5}$ Members were recruited via the PML and UT researchers' networks.
} 
To account for the quality of the literature review, interviews and FGs, we applied 'ENhancing Transparency in REporting the synthesis of Qualitative research' (ENTREQ) [37] (see: https://osf.io/b2yeh/); and 'COnsolidated criteria for Reporting Qualitative research (COREQ; Tong 2011) [38] (see: https://osf.io/wdjax/). Triangulation was based on a variety of researchers; various data sources (also reflecting diverse stakeholders); comprehensive data analysis through open coding; and iterative axial coding, and data synthesis [36, 39, 40]. Our main data sources comprised: literature; interviews' and FGs. We deliberately choose not to include, in these date sources, literature or expertise from outside the Netherlands. Since our objective was to develop a national ML framework (i.e., contextually appropriate to the characteristics of the Dutch culture, health system, healthcare field and its professions) we exclusively used Dutch (oriented) publications and sought for interviewees and participants working in Dutch healthcare (organizations). In a final phase, we did however compare a pre-final version of the framework with existing non-Dutch frameworks.

To ensure high validity of our analysis we deployed: individual data analysis by researchers; iterative crosschecking of results and open plenary discussions and consensus procedures; structured debriefing; audit trailing and logging; and nonvoting researchers: to expedite consensus forming or to resolve slight differences (WK or CW) [41-44].

\section{Literature review}

To assure appropriate data interpretation and optimal reflection of the relevant needs in the Dutch healthcare system, and to focus on outcome abilities, we chose to include both 'white' as well as 'grey' literature in our review [21, 45]. Following the guidelines for Cochrane Reviews, in- and exclusion criteria for 'white' sources and defined search terms were determined (Table 2) $[45,46]$. To validate accuracy, the search strategy was verified with a
Table 2 In- and exclusion criteria for literature selection

\begin{tabular}{|c|c|}
\hline nclus & Exclusion criteria \\
\hline $\begin{array}{l}\text { - Concerns or has generalizable } \\
\text { relevance to Dutch medical } \\
\text { sector } \\
\text { - Relates to the 'leadership' concept } \\
\text { (involving behavior / personality } \\
\text { traits / attitude / roles / tasks; not } \\
\text { just related to financial or } \\
\text { organizational structures or } \\
\text { management contexts) }\end{array}$ & $\begin{array}{l}\text { - Individual patient care } \\
\text { - Clinical work } \\
\text { - ML only in Conclusion or } \\
\text { Discussion sections } \\
\text { - Evaluation of cost-effectiveness } \\
\text { of therapies } \\
\text { - Non-Dutch context related studies } \\
\text { - Publication date }<2004\end{array}$ \\
\hline
\end{tabular}

${ }^{\mathrm{a} N o t}$ explicating $\mathrm{ML}$ or related concepts

similar prior review [13]. We applied a sensitivitymaximizing approach using EMBASE and MEDLINE data bases [45]. 'Grey' literature included records retrieved from: researchers' private libraries; consultations with topic experts; databases of relevant websites (e.g., government policy reports; medical association database); and online (GoogleScholar ${ }^{\mathrm{ru}}$ ) searches, using various search terms (see: https://osf. io/kh2vx/). Inclusion-exclusion analysis resulted in a total of 67 records that were coded (Fig. 3). One Flemish paper was deemed generalizable to the Dutch context [47*] (Tables 3 and 4). The 26 included 'white' records reflected five fields: improvement and innovation (8); training and education (6); administration and policy issues (5); integrated care and multi-disciplinary disease management (4); and human resources (3). The heterogeneity of the included 'grey' records' content disallowed similar categorization.

The researchers assessed, in pairs, all the records' titles and abstracts for eligibility; after an individual pre-assessment, both researchers convened for a discussion, and eventually reached a consensus on the initial 'white' literature inclusions. A review of a selection of included papers by selected international topic experts confirmed the search accuracy. Full-text eligibility was also assessed in pairs. 'Grey' literature inclusion followed a similar eligibility process. To increase sensitivity, in- and exclusion criteria were adjusted

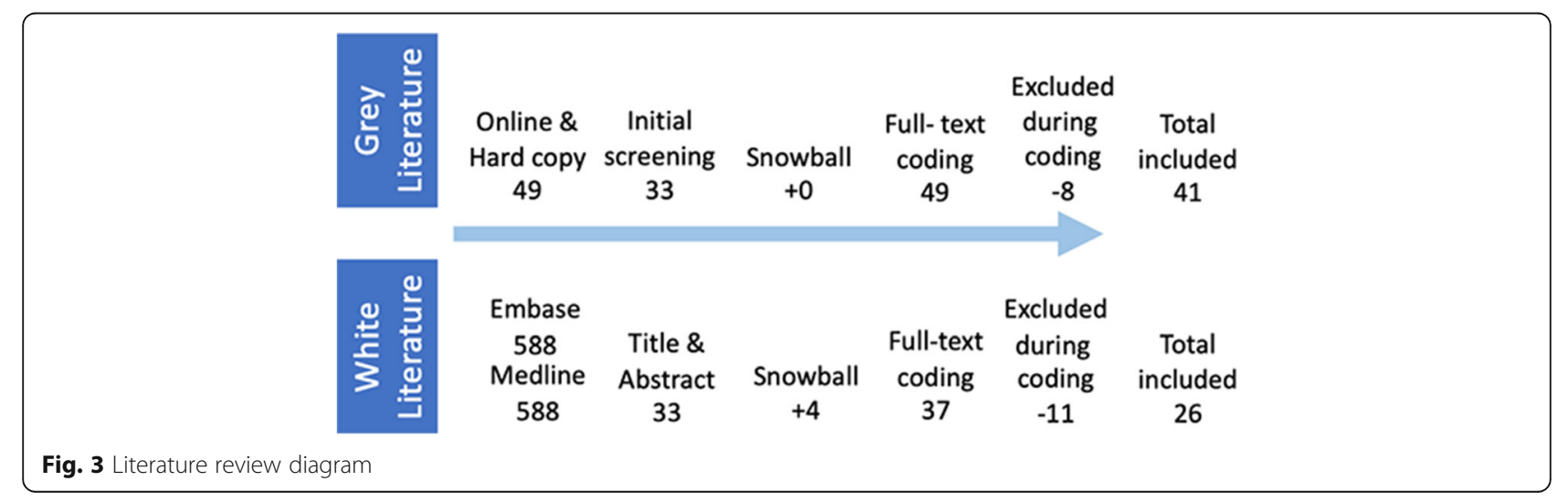


Table 3 Characteristics of the included 'white' literature

\begin{tabular}{|c|c|c|c|c|}
\hline $\begin{array}{l}\text { 1st author, } \\
\text { publication } \\
\text { year } \\
\text { (nationality) } \\
\text { (category }^{a} \text { ) }\end{array}$ & Article type / Method & Objective & Focus & Relevant findings \\
\hline $\begin{array}{l}\text { 1. Fleuren, } \\
2004\left[48^{*}\right] \\
\text { (Dutch) (1) }\end{array}$ & $\begin{array}{l}\text { Literature study and Delphi } \\
\text { consultation }\end{array}$ & $\begin{array}{l}\text { Validate determinants of } \\
\text { innovations with Dutch } \\
\text { implementation experts }\end{array}$ & $\begin{array}{l}\text { Innovations in large } \\
\text { healthcare systems }\end{array}$ & $\begin{array}{l}\text { Impact of opinion-leadership on } \\
\text { innovation }\end{array}$ \\
\hline $\begin{array}{l}\text { 2. Bloemen, } \\
2005\left[49^{*}\right] \\
\text { (Dutch) (4) }\end{array}$ & $\begin{array}{l}\text { Model development and } \\
\text { evaluation; mixed methods }\end{array}$ & $\begin{array}{l}\text { Study enabling factors and barriers } \\
\text { for implementation transmural care } \\
\text { in a Dutch region }\end{array}$ & $\begin{array}{l}\text { Transmural care model } \\
\text { implementation }\end{array}$ & $\begin{array}{l}\text { Individual professional's (eagerness for) } \\
\text { learning knowledge, skills and } \\
\text { competencies for transmural care }\end{array}$ \\
\hline $\begin{array}{l}\text { 3. Scholten, } \\
2005\left[50^{*}\right] \\
\text { (Dutch) (3) }\end{array}$ & $\begin{array}{l}\text { Mixed methods: document } \\
\text { analysis and semi-open } \\
\text { interviews }\end{array}$ & $\begin{array}{l}\text { Study of executives' and medical } \\
\text { staff's role in medical governance } \\
\text { in Dutch hospitals }\end{array}$ & $\begin{array}{l}\text { Policy implementation } \\
\text { and effects of collective } \\
\text { counteractivities of } \\
\text { physicians }\end{array}$ & $\begin{array}{l}\text { Challenges of and role of physicians in } \\
\text { 'medical governance' in hospitals }\end{array}$ \\
\hline $\begin{array}{l}\text { 4. Prince, } 2005 \\
{\left[51^{*}\right] \text { (Dutch) }} \\
\text { (2) }\end{array}$ & $\begin{array}{l}18 \text { months post-graduate } \\
\text { evaluation of problem- } \\
\text { based learning (PBL) re. } \\
\text { general competencies }\end{array}$ & $\begin{array}{l}\text { Compare PBL versus non-PBL } \\
\text { among Dutch junior doctors }\end{array}$ & $\begin{array}{l}\text { General educational } \\
\text { competencies }\end{array}$ & $\begin{array}{l}\text { PBL possibly preferable for some } \\
\text { competencies }\end{array}$ \\
\hline $\begin{array}{l}\text { 5. Van Raak, } \\
2008\left[52^{*}\right] \\
\text { (Dutch) (4) }\end{array}$ & Case study; mixed methods & $\begin{array}{l}\text { Study routines and cooperation in } \\
\text { Dutch regional integrated care }\end{array}$ & $\begin{array}{l}\text { Disparate matches } \\
\text { between professional } \\
\text { routines }\end{array}$ & $\begin{array}{l}\text { (Transformational) leadership can } \\
\text { facilitate routine divergence }\end{array}$ \\
\hline $\begin{array}{l}\text { 6. Duckers, } \\
2009\left[53^{*}\right] \\
\text { (Dutch) }(1)\end{array}$ & $\begin{array}{l}\text { Multilevel analysis } \\
\text { (physician data) }\end{array}$ & $\begin{array}{l}\text { Study effect of leadership on } \\
\text { participation in improvement } \\
\text { programs }\end{array}$ & $\begin{array}{l}\text { Leadership climate } \\
\text { influencing (physician) } \\
\text { engagement in } \\
\text { innovation Dutch } \\
\text { hospitals }\end{array}$ & $\begin{array}{l}\text { Importance of leadership visibility and } \\
\text { minimizing ambiguity on leadership } \\
\text { intentions }\end{array}$ \\
\hline $\begin{array}{l}\text { 7. Klopper, } \\
2009\left[54^{*}\right] \\
\text { (Dutch) (3) }\end{array}$ & Mixed methods & $\begin{array}{l}\text { Study of relative status, power, and } \\
\text { goal incompatibility }\end{array}$ & $\begin{array}{l}\text { Image Theory in Dutch } \\
\text { physician-manager } \\
\text { relationship }\end{array}$ & $\begin{array}{l}\text { Need for physicians to understand } \\
\text { management perspective }\end{array}$ \\
\hline $\begin{array}{l}8 . \\
\text { Berkenbosch, } \\
2011\left[55^{*}\right] \\
\text { (Dutch) (2) }\end{array}$ & Questionnaire & $\begin{array}{l}\text { Study of residents' perceptions and } \\
\text { understanding of management } \\
\text { skills and knowledge }\end{array}$ & $\begin{array}{l}\text { Management } \\
\text { competency training for } \\
\text { Dutch physicians }\end{array}$ & $\begin{array}{l}\text { Management competency training for } \\
\text { junior physicians needs improvement }\end{array}$ \\
\hline $\begin{array}{l}\text { 9. Cramm, } \\
2011\left[56^{*}\right] \\
\text { (Dutch) (4) }\end{array}$ & $\begin{array}{l}\text { Validity and reliability } \\
\text { (psychometric) testing }\end{array}$ & $\begin{array}{l}\text { Validate Partnership Self- } \\
\text { Assessment Tool (PSAT) in Dutch } \\
\text { chronic care }\end{array}$ & $\begin{array}{l}\text { Professional partnership } \\
\text { synergy in disease } \\
\text { management }\end{array}$ & $\begin{array}{l}\text { Leadership competencies influence } \\
\text { partnership functioning }\end{array}$ \\
\hline $\begin{array}{l}\text { 10. Klopper, } \\
2011\left[57^{*}\right] \\
\text { (Dutch) (3) }\end{array}$ & Semi-structured interviews & $\begin{array}{l}\text { Study on influence of Dutch } \\
\text { manager-physician and managers } \\
\text { cooperation on hospital } \\
\text { performance }\end{array}$ & $\begin{array}{l}\text { Intergroup conflict } \\
\text { theory and manager- } \\
\text { physician cooperation }\end{array}$ & $\begin{array}{l}\text { Medical-management culture } \\
\text { influence, intra-hospital cooperation } \\
\text { and performance }\end{array}$ \\
\hline $\begin{array}{l}\text { 11.Schreuder, } \\
2011\left[58^{*}\right] \\
\text { (Dutch) (5) }\end{array}$ & Cross-sectional study & $\begin{array}{l}\text { Investigation of leadership-sickness } \\
\text { absence relationship }\end{array}$ & $\begin{array}{l}\text { Leadership styles and } \\
\text { sickness absence in } \\
\text { Dutch healthcare }\end{array}$ & $\begin{array}{l}\text { Relationship-oriented leadership styles } \\
\text { can facilitate efficiency and quality }\end{array}$ \\
\hline $\begin{array}{l}\text { 12. Teunissen, } \\
2011\left[59^{*}\right] \\
\text { (Dutch) (2) }\end{array}$ & $\begin{array}{l}\text { Medical education related } \\
\text { commentary }\end{array}$ & Editorial comment on publications & $\begin{array}{l}\text { Transition from } \\
\text { 'learning' to } \\
\text { 'performing' }\end{array}$ & $\begin{array}{l}\text { Metacognitive skills can facilitate entry } \\
\text { into medical practice }\end{array}$ \\
\hline $\begin{array}{l}\text { 13. Van der } \\
\text { Lee, } 2011\left[60^{*}\right] \\
\text { (Dutch) (2) }\end{array}$ & $\begin{array}{l}\text { Inductive analysis of semi- } \\
\text { structured open-ended } \\
\text { questionnaire }\end{array}$ & $\begin{array}{l}\text { To test content validity of } \\
\text { CanMEDS framework }\end{array}$ & $\begin{array}{l}\text { Dutch physicians' vision } \\
\text { of future generic } \\
\text { medical competencies }\end{array}$ & $\begin{array}{l}\text { Curriculum design could benefit from } \\
\text { (strategically planned) external } \\
\text { influences }\end{array}$ \\
\hline $\begin{array}{l}\text { 14. Berben, } \\
2012\left[61^{*}\right] \\
\text { (Dutch) (4) }\end{array}$ & $\begin{array}{l}\text { Qualitative: focus groups } \\
\text { and interviews }\end{array}$ & $\begin{array}{l}\text { Identification of determinants in } \\
\text { pain management in Dutch } \\
\text { emergency care }\end{array}$ & $\begin{array}{l}\text { Changing protocols in } \\
\text { care chains }\end{array}$ & $\begin{array}{l}\text { (Physician) role modelling can } \\
\text { facilitate professional communication } \\
\text { and attitude }\end{array}$ \\
\hline $\begin{array}{l}\text { 15. Buljac, } \\
\text { 2012 [62*] } \\
\text { (Dutch) (1) }\end{array}$ & $\begin{array}{l}\text { Cross-sectional survey in } \\
\text { Dutch long-term care }\end{array}$ & $\begin{array}{l}\text { Impact of team member stability, } \\
\text { team coaching, and error } \\
\text { orientation on team safety and } \\
\text { innovation }\end{array}$ & $\begin{array}{l}\text { Team safety and } \\
\text { innovation in long-term } \\
\text { care teams }\end{array}$ & $\begin{array}{l}\text { (Team) coaching leadership styles is } \\
\text { related to stability and safety of care }\end{array}$ \\
\hline $\begin{array}{l}\text { 16. Ovretveit, } \\
2012\left[63^{*}\right] \\
\text { (Swedish/ } \\
\text { Dutch) (1) }\end{array}$ & Mixed-methods comparison & $\begin{array}{l}\text { Evaluation of large-scale Dutch } \\
\text { health and social care improvement } \\
\text { programs }\end{array}$ & $\begin{array}{l}\text { Success of national } \\
\text { improvement initiatives }\end{array}$ & $\begin{array}{l}\text { Clinical championing affects } \\
\text { implementation success of } \\
\text { improvement programs }\end{array}$ \\
\hline
\end{tabular}


Table 3 Characteristics of the included 'white' literature (Continued)

\begin{tabular}{|c|c|c|c|c|}
\hline $\begin{array}{l}\text { 1st author, } \\
\text { publication } \\
\text { year } \\
\text { (nationality) } \\
\left(\text { category }^{\mathrm{a}}\right)\end{array}$ & Article type / Method & Objective & Focus & Relevant findings \\
\hline $\begin{array}{l}\text { 17. Smith, } \\
2012\left[64^{*}\right] \\
\text { (international) } \\
\text { (3) }\end{array}$ & Structured survey & $\begin{array}{l}\text { Governance arrangements in } \\
\text { leadership and healthcare in } \\
\text { developed countries }\end{array}$ & $\begin{array}{l}\text { Leadership, governance } \\
\text { and accountability in } \\
\text { health systems }\end{array}$ & $\begin{array}{l}\text { Awareness raising of national } \\
\text { healthcare priority setting and } \\
\text { performance indicators and } \\
\text { monitoring }\end{array}$ \\
\hline $\begin{array}{l}\text { 18. Van Daele, } \\
2012\left[47^{*}\right] \\
\text { (Flemish) (3) }\end{array}$ & Symposium abstract & $\begin{array}{l}\text { Conflicting priorities within } \\
\text { responsibilities of clinical leaders, } \\
\text { vis-a-vis management, staff and } \\
\text { patients }\end{array}$ & $\begin{array}{l}\text { Role of clinical } \\
\text { department leaders }\end{array}$ & $\begin{array}{l}\text { Conflicting priorities in clinical } \\
\text { leadership and management roles can } \\
\text { create vulnerability }\end{array}$ \\
\hline $\begin{array}{l}\text { 19. Aij, } 2013 \\
\text { [65*] (Dutch) } \\
\text { (1) }\end{array}$ & $\begin{array}{l}\text { Semi-structured, in-depth } \\
\text { interviews in Dutch } \\
\text { hospitals }\end{array}$ & $\begin{array}{l}\text { Determinants of lean } \\
\text { implementation from a leadership } \\
\text { perspective }\end{array}$ & $\begin{array}{l}\text { Lean improvement } \\
\text { implementation }\end{array}$ & $\begin{array}{l}\text { Leadership (competencies like) role } \\
\text { modelling, visibility and vision across } \\
\text { multidisciplinary shared learning } \\
\text { facilitates lean implementation }\end{array}$ \\
\hline $\begin{array}{l}20 . \\
\text { Berkenbosch, } \\
2013\left[66^{*}\right] \\
\text { (Dutch) (2) }\end{array}$ & $\begin{array}{l}\text { Online survey to Dutch } \\
\text { medical specialists }\end{array}$ & $\begin{array}{l}\text { Need for management training } \\
\text { among Dutch residents }\end{array}$ & $\begin{array}{l}\text { Manager competency } \\
\text { training to residents }\end{array}$ & $\begin{array}{l}\text { Management competency education } \\
\text { should entail leadership skills }\end{array}$ \\
\hline $\begin{array}{l}\text { 21. Cramm, } \\
\text { 2013a }\left[67^{*}\right] \\
\text { (Dutch) (1) }\end{array}$ & $\begin{array}{l}\text { Cross-sectional survey in } \\
\text { Dutch long-term care }\end{array}$ & $\begin{array}{l}\text { Investigation of partnership synergy } \\
\text { during innovations }\end{array}$ & $\begin{array}{l}\text { Sustainability of } \\
\text { innovations in } \\
\text { community care } \\
\text { settings }\end{array}$ & $\begin{array}{l}\text { Leadership competencies, in relation } \\
\text { to 'boundary spanning', benefit } \\
\text { sustainability of innovations }\end{array}$ \\
\hline $\begin{array}{l}\text { 22. Cramm, } \\
\text { 2013b }\left[68^{*}\right] \\
\text { (Dutch) }(5)\end{array}$ & $\begin{array}{l}\text { Cross-sectional survey in } \\
\text { Dutch long-term care }\end{array}$ & $\begin{array}{l}\text { Organizational characteristics } \\
\text { related to employee solidarity }\end{array}$ & $\begin{array}{l}\text { Effect of employee } \\
\text { solidarity on } \\
\text { effectiveness and } \\
\text { efficiency }\end{array}$ & $\begin{array}{l}\text { Transformational leadership styles } \\
\text { enhance employee solidarity }\end{array}$ \\
\hline $\begin{array}{l}\text { 23. Elshout, } \\
2013\left[69^{*}\right] \\
\text { (Dutch) (5) }\end{array}$ & $\begin{array}{l}\text { Mixed methods design: } \\
\text { interviews and document } \\
\text { study }\end{array}$ & $\begin{array}{l}\text { Investigation of association } \\
\text { between leadership style, } \\
\text { absenteeism, and employee } \\
\text { satisfaction in mental health care } \\
\text { institutions }\end{array}$ & $\begin{array}{l}\text { Leadership style, } \\
\text { employee satisfaction } \\
\text { and absenteeism }\end{array}$ & $\begin{array}{l}\text { Transformational leadership benefits } \\
\text { employee satisfaction and } \\
\text { absenteeism }\end{array}$ \\
\hline $\begin{array}{l}\text { 24. Huis, } 2013 \\
\text { [70*] (Dutch) } \\
\text { (1) }\end{array}$ & $\begin{array}{l}\text { Process evaluation of a } \\
\text { randomized controlled trial }\end{array}$ & $\begin{array}{l}\text { Association between hand hygiene } \\
\text { improvement strategies and } \\
\text { compliance }\end{array}$ & $\begin{array}{l}\text { Quality improvement } \\
\text { strategies }\end{array}$ & $\begin{array}{l}\text { Effects of team leadership and role } \\
\text { modelling on hygiene compliance }\end{array}$ \\
\hline $\begin{array}{l}\text { 25. ljkema, } \\
2013\left[71^{*}\right] \\
\text { (Dutch) (1) }\end{array}$ & $\begin{array}{l}\text { Semi-structured interviews } \\
\text { in Dutch hospitals }\end{array}$ & $\begin{array}{l}\text { Identification of determinants for } \\
\text { successful implementation } \\
\text { improvement initiative }\end{array}$ & $\begin{array}{l}\text { Implementation of } \\
\text { complex multi- } \\
\text { component improve- } \\
\text { ment programs }\end{array}$ & $\begin{array}{l}\text { Importance of effective leadership in } \\
\text { project management }\end{array}$ \\
\hline $\begin{array}{l}\text { 26. Witman, } \\
2013\left[72^{*}\right] \\
\text { (Dutch) (2) }\end{array}$ & Descriptive case study & Report of a pilot study & $\begin{array}{l}\text { Professional identity } \\
\text { and education in } \\
\text { reflective practice }\end{array}$ & $\begin{array}{l}\text { Reflection on practices: Balancing } \\
\text { between conflicting responsibilities }\end{array}$ \\
\hline
\end{tabular}

${ }^{a}$ Category: (1) improvement and innovation; (2) training and education; (3) administration and policy issues; (4) integrated care and multidisciplinary disease management; and (5) human resources

based on initial findings: a process called 'niche shaping $^{6}$ [73]. During this process of fine-tuning criteria, it became apparent that publications mentioning 'leadership' (or related search terms), often entailed studies on clinical enquiries, not explicating meaning or use of $\mathrm{ML}$ in any form, resulting in the final set of criteria. Backward citations or 'snowball' searches were performed on all the included 'white' and 'grey' records to complete the search.

\footnotetext{
${ }^{6}$ Apart from finding some additional nursing leadership articles, niche shaping did not result in additional records.
}

To limit inter-coder bias and to increase reliability, subsequent open coding was also done by the researcher pairs. They analyzed all the included literature, first individually, then by convening to discuss: intermediate results; definition or adjustment of coding terms; and eventual consensus. Coded text fragments were recorded in a data base (Microsoft ${ }^{\text {tix }}$ Excel) based on the data extraction questions and quality using: a) an adapted version of the JBI-QARI quality checklist [74]; and b) the American Association of Critical Care Nursing levels of evidence [75] (see: https://osf.io/r8ucj/).

Although none of the included records disclosed explicit descriptions of ML competencies or an explicit definition 
Table 4 Characteristics included in the 'grey' literature

\begin{tabular}{lll}
\hline Record type & Total of records & $\%$ \\
\hline 1. Online web pages & 11 & $26.8 \%$ \\
2. Opinion article & 6 & $14.6 \%$ \\
3. Journalistic article & 6 & $14.6 \%$ \\
4. Professional association paper / report & 4 & $9.8 \%$ \\
5. Thesis (MSc or PhD) & 4 & $9.8 \%$ \\
6. Professional journal (not indexed) & 3 & $7.3 \%$ \\
7. Book chapter & 2 & $4.9 \%$ \\
8. Essay & 2 & $4.9 \%$ \\
9. Policy (research) report & 2 & $4.9 \%$ \\
10. Healthcare organization report & 1 & $2.4 \%$ \\
Total records & 41 & $100.0 \%$ \\
\hline
\end{tabular}

of ML, they all provided features of ML's concept. Eventually, during three interactive sessions and using visual materials (cards with quotations, representing codes), we performed axial coding, and iteratively composed sets of interrelating codes, categorizing the 208 coded fragments into 14 competence themes (Table 5).

\section{Field interviews}

Semi-structured explorative interviews were held [76]. Thirty-five persons were invited, representing two stakeholder groups; 33 persons agreed to participate in the interviews ( 2 interviews were discarded: see below) (Table 6). The first group comprised Dutch medical

Table 5 Medical leadership themes from axial coding of literature

\begin{tabular}{lll}
\hline Literature & & \\
\hline Theme & $\begin{array}{l}\text { Total coded } \\
\text { fragments }\end{array}$ & Percentage \\
\hline 1. Collaboration & 37 & $17.9 \%$ \\
2. Coach and guide & 31 & $15.0 \%$ \\
3. Personal development & 26 & $12.6 \%$ \\
4. Organize & 16 & $7.7 \%$ \\
5. Quality improvement & 15 & $7.2 \%$ \\
6. Role modelling and visibility & 14 & $6.8 \%$ \\
7. Responsibility \& decision making & 12 & $5.8 \%$ \\
8. Entrepreneurship & 11 & $5.3 \%$ \\
9. Vision & 11 & $5.3 \%$ \\
10. Resources management & 9 & $4.3 \%$ \\
11. Integrity & 7 & $3.4 \%$ \\
12. Managerial / governance & 7 & $3.4 \%$ \\
13. Patient centered & 7 & $3.4 \%$ \\
14. Communication & 4 & $1.9 \%$ \\
Total fragments white and grey & 208 & $100.0 \%$ \\
literature & & \\
\hline
\end{tabular}

professionals $(n=21)$ across the practice domains of hospital, primary, public health and social care, including three medical students. These interviewees were identified from various networks linked to the 14 researchers, including the PML member data base. The second group encompassed $(n=10)$ non-medical interviewees from: allied healthcare professions; healthcare management; the Dutch Patient Federation and KNMG. These interviewees were selected by contacting the noted organizations which provided two representatives each. Eligibility for inviting interviewees was based on creating a balanced heterogeneity in medical practice domains (first group), and other stakeholders in Dutch healthcare (second group). None of the interviewees had been involved specifically in prior (national) ML development activities or related research.

An open-ended questions' protocol was made after studying the extant literature and reports on existing ML frameworks (e.g.: [5, 20, 77-79]) (see: https://osf. io/m93yq/). To enhance the interviewers' neutral position towards interview topics, and to minimize subjectivity (e.g., 'Heisenberg Effect') [80], all (nine) researchers who performed the interviews were briefed, using detailed instructions. Interviews were conducted preferably face-to-face, in a quiet place to diminish disturbances, recorded and transcribed verbatim (anonymized) [41, 80]. The interviewees' consent to use the interview's anonymized information for our study was provided before the start of each interview. All interviews lasted between 40 and 75 min; six interviews $(23 \%)$ were held via telephone or Skype ${ }^{\mathrm{m}}$. Two interviews were discarded (recording malfunctioning) and two were cancelled due to logistics, resulting in 31 interviews for analysis, thus remaining within recommended boundaries [81].

Interview transcript analysis involved semi-open coding with analytic software (ATLAS.ti, Scientific Software Development $\mathrm{GmbH}, 2012$ ). Three researchers developed an initial coding list of 47 labels by independently screening a randomly selected sample of three transcripts, and subsequent discussions. Then, the list was tested by individually coding a fourth randomly selected transcript, revealing a satisfactory $90 \%$ inter-coder correspondence and resulting in two new labels. Hereafter, six researchers independently coded all the remaining transcripts in pairs, before openly discussing the results in pairs. After coding interview number 29, no new labels were identified, indicating 'saturation' [82]. One thousand three hundred ninetysix interview fragments were digitally collected and categorized over 67 distinct labels. Finally, on applying axial coding during a final researchers' meeting all 67 labels were thematically distributed into 9 distinct overarching themes (Table 7). 
Table 6 Characteristics interviews participants

\begin{tabular}{|c|c|c|c|}
\hline Medical Interviewees & $N=21$ & Non-Medical Interviewees & $N=10$ \\
\hline$\%$ Male & $57.1 \%$ & $\%$ Male & $70 \%$ \\
\hline$\%$ Female & $42.9 \%$ & $\%$ Female & $30 \%$ \\
\hline Average age & $42.7 \mathrm{yrs}$ & Average age & $51.2 \mathrm{yrs}$. \\
\hline Hospital care & $N=6$ & Para-medical & $N=2$ \\
\hline - Average age & 35.5 yrs. & - Average age & $47.5 \mathrm{yrs}$. \\
\hline • \% male & $50 \%$ & $\cdot \%$ male & $0 \%$ \\
\hline • \% female & $50 \%$ & • \% female & $100 \%$ \\
\hline Primary care & $N=6$ & Patient association representatives & $N=2$ \\
\hline - Average age & $49.5 \mathrm{yrs}$ & - Average age & $53.5 \mathrm{yrs}$. \\
\hline • \% male & $53.3 \%$ & • \% male & $50 \%$ \\
\hline • \% female & $16.7 \%$ & • \% female & $50 \%$ \\
\hline Social care & $N=6$ & Hospital administrators & $N=2$ \\
\hline - Average age & $51.6 \mathrm{yrs}$ & - Average age & $42.5 \mathrm{yrs}$. \\
\hline • \% male & $66.6 \%$ & • \% male & $100 \%$ \\
\hline • \% female & $33.3 \%$ & • \% female & $0 \%$ \\
\hline Medical students & $N=3$ & Managers & $N=2$ \\
\hline - Average age & $25.6 \mathrm{yrs}$ & - Average age & $51.5 \mathrm{yrs}$. \\
\hline • \% male & $0 \%$ & • \% male & $100 \%$ \\
\hline \multirow[t]{5}{*}{ • \% female } & $100 \%$ & • \% female & $0 \%$ \\
\hline & & Professional association representatives & $N=2$ \\
\hline & & - Average age & 61.0 yrs. \\
\hline & & • \% male & $100 \%$ \\
\hline & & • \% female & $0 \%$ \\
\hline
\end{tabular}

\section{Synthesis version 0.1}

The literature synthesis and interviews were guided by Grounded Theory [36, 73, 83]. On discussing the initial analysis of the results, we decided to value the coded data from the literature and interviews as equals, and did not discriminate on, for example, the coding frequency. Then, while iteratively discussing the intermediate results during

Table 7 Medical leadership themes from axial coding of interviews

\begin{tabular}{lll}
\hline Interviews & & \\
\hline Theme & Total coded fragments & Percentage \\
\hline 1. Collaborate & 362 & $25.9 \%$ \\
2. Organize & 273 & $19.6 \%$ \\
3. Coaching & 145 & $10.4 \%$ \\
4. Self-reflection & 137 & $9.8 \%$ \\
5. Responsibility & 120 & $8.6 \%$ \\
6. Future perspective & 108 & $7.7 \%$ \\
7. Quality & 105 & $7.5 \%$ \\
8. Decision making & 90 & $6.4 \%$ \\
9. Societal contract & 56 & $4.0 \%$ \\
& 1396 & $100.0 \%$ \\
\hline
\end{tabular}

three sessions, we combined all the identified categories and themes into more homogeneous interpretable thematic groups. Next, based on this new collection of categories and their underlying content (i.e., coded fragments), an initial conceptual version of the framework was drafted by one researcher (WK). This was done to assure that all the themes identified from both the literature and interviews were accounted for as well as retrievable in the text. Subsequently, based on the initial draft, a version, the 0.1 version of the DML framework was designed by a subgroup of five researchers after a process of iterative discussing and intermittent editing of successive versions of the initial draft. During this process, whilst continuously consulting the original data, the researchers documented their comments and issues using online shared Excel ${ }^{\mathrm{Tm}}$ forms for cross-checking.

Parallel to this, another subgroup systematically analyzed all the included literature and transcripts, selecting relevant fragments to compose an abstract definition of the ML concept, using analytic software (ATLAS.ti ${ }^{\mathrm{TM}}$ ). After individually coding fragments of components describing ML, its concept, or distinct competencies, three of the core researchers reached a consensus on the prefinal ML definition. 


\section{International comparison}

To validate completeness and to search for relevant (e.g., inter-cultural) differences, a subgroup reviewed foreign ML frameworks (e.g., [3, 19, 77, 84, 85]), and provided their findings to the core group. Although this comparison did not reveal new ML-related themes or domains, it aided the researchers with more nuances to word the resulting 0.2 version, which was then used for facevalidity testing.

\section{Validation of version 0.2}

Face-validity testing of version 0.2 of the DML framework was done through an online survey and three FG discussions. After an open invitation to all PML members (February 2015), 52 persons (comprising approximately $25 \%$ of PML's membership) volunteered to participate in a FG. Based on the availability for the planned dates, 42 were invited, and eventually 27 participated (35.7\%, due to no-shows or late cancellations). Prior to each session, all participants received, per email, version 0.2 of the DML framework and a concise agenda of the FG session. One researcher facilitated the sessions (WK), using a topic list, by following a loose interactive structure, thereby allowing ample discussion; one researcher observed and took notes. Consent was collected from the participants at the start of each session, which lasted between $110 \mathrm{~min}$ to $2 \mathrm{~h}$ and was recorded and transcribed verbatim (anonymized). Notes were compared during the research debriefing immediately after each session [44].

An online survey (SurveyMonkey ${ }^{\mathrm{Tm}}$ ) was created to validate the 0.2 DML framework version, including the definition for ML, using a 5-point Likert scale as well as open questions [85]. The survey was sent to 142 individuals, including: PML members who had applied for FGs $(n=52)$; past interviewees $(n=32) \quad$ ('member check' [86]); and a convenience sample of other PML members $(n=68)$ (Table 8).

Table 8 Response validity survey $(n=82)$

\begin{tabular}{llll}
\hline $\begin{array}{l}\text { Response } \\
\text { group }\end{array}$ & $\begin{array}{l}\text { Invited } \\
\text { individuals }\end{array}$ & $\begin{array}{l}\text { Number of } \\
\text { Respondents }\end{array}$ & $\begin{array}{l}\text { Response rate } \\
(\%)\end{array}$ \\
\hline $\begin{array}{l}\text { Focus group } \\
\# 1\end{array}$ & 10 & 8 & $80.0 \%$ \\
$\begin{array}{l}\text { Focus group } \\
\# 2\end{array}$ & 15 & 14 & $93.3 \%$ \\
$\begin{array}{l}\text { Focus group } \\
\text { \#3 }\end{array}$ & 17 & 10 & $58.8 \%$ \\
$\begin{array}{l}\text { Interviewees } \\
\text { PML }\end{array}$ & $32^{\mathrm{a}}$ & 12 & $37.5 \%$ \\
members & 68 & 38 & $55.9 \%$ \\
Total & 142 & 82 & $65.1 \%$ \\
\hline
\end{tabular}

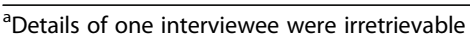

The survey respondents $(n=82)$ represented various professional domains: family practitioners (32.5\%); medical specialists (21.3\%); non-clinical respondents (management; patient and professional associations; etc.) (27.5\%); and medical students and interns (18.8\%) (response rate: 65\%; female-male ratio: $30 / 70 \%$; average age: 40 years). The survey involved rating all the DML framework (version 0.2) domains in terms of recognition of the relevant value of the current practice $^{7}$ Respondents also offered written feedback on other (open) questions. Survey outcomes were stored on worksheets (Microsoft Excel $\left.{ }^{\mathrm{m}}\right)$ and analyzed using SPSS $^{\mathrm{mw}}$.

\section{English translation}

To ensure cultural integrity after completing version 1.0 (see Results section), four researchers took a threepronged approach to translate the final 1.0 DML framework version into English (see: https://osf.io/qknds/). This comprised various sessions based on: (1) professional translation services (NEN-EN 15038 certified); (2) topicalexpert translation; and (3) backward translation [87].

\section{Results}

The foregoing details the various phases and activities during the framework's development. Below we elaborate on the resulting 1.0 DML framework.

\section{Final version}

The framework's final version used feedback from testing of version 0.2 . The analysis of FG transcripts and the survey data did not provide new elements of ML, indicating a relatively high level of completeness. Yet, FG transcripts and survey data revealed that version 0.2 was not seen as completely sufficient. Survey respondents appreciated the initiative of creating a national framework with a relatively satisfactory score: $7.6 / 10$ (SD 1.37) (Fig. 4). Correspondingly, the perceived relevance criteria scores of the $12 \mathrm{ML}$ competency domains were rated relatively high in the surveys (Fig. 5), concurring with notions found in the FG transcripts. However, the content of version 0.2 was rated slightly lower $(6.8 / 10$; SD 1.42). Also, the survey respondents described the content as overly 'wordy' and long, which concurred with the descriptions in the FG transcripts. Thus, it was concluded that there was a need for improvement in the usability of version 0.2 in terms of: conciseness; clarity; and readability.

Face-validity concerns instigated a final round of textual editing of version 0.2. Superfluous and repetitive items were removed. Version 0.2 was refined to a more

${ }^{7}$ Practical value indicators: 'acknowledgment'; 'completeness'; 'clarity'; 'readability'; and 'relevance' to: (1) healthcare in general; (2) continuing medical education; (3) medical training; and (4) respondent's specialty. 


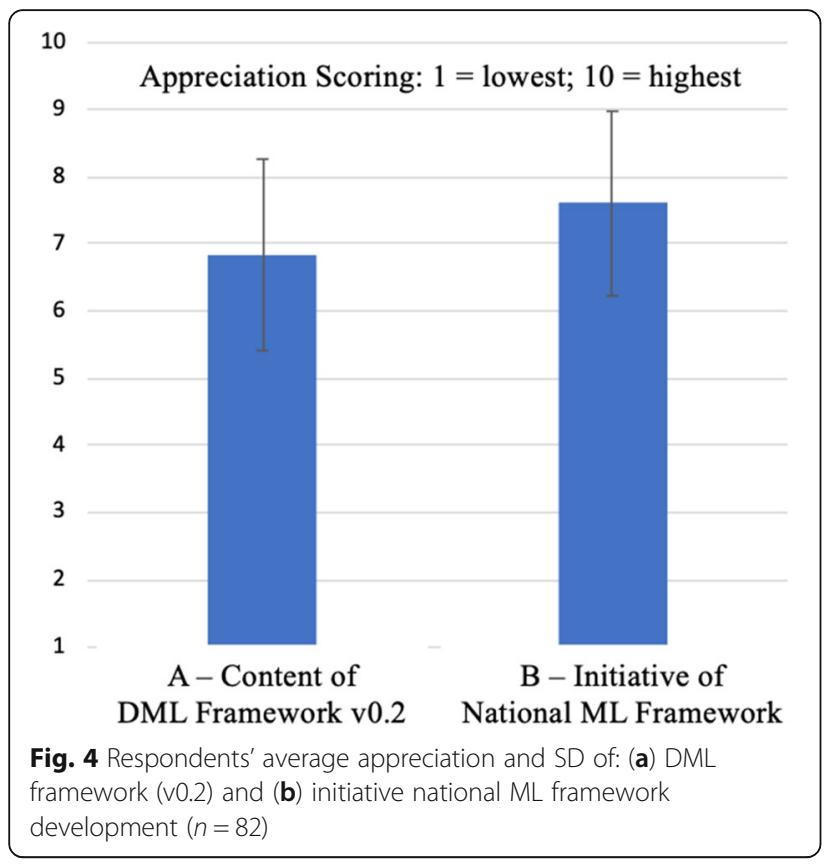

concise and less abstract version. It was shortened from 1890 to 1290 words, and competency items per domain were reduced by nearly 60\% (from an average of 7 to 4 items per domain). The result was version 1.0.

Eventually, based on selective individual coding, during a final consensus session, the core group members constructed a graphical representation of any interrelations between the domains and three overarching dimensions: 'Me'; 'Others'; and 'Society'
[88]. The final version consisted of 12 domains, each entailing 4 distinct competencies and a compact ML definition (see: https://osf.io/qknds/) (Fig. 4).

\section{Discussion}

In this section, we reflect on our findings in the face of current scholarly understandings. First, we describe, from our frameworks' perspective, the changing nature of 'the' physician. Next, we reflect on possible uses of our study's results, and then discuss the study's strengths and limitations. We close with suggestions for related future research.

\section{The twenty-first century physician}

The three dimensions encompassing the 12 ML domains and their competences (Fig. 6) correspond with extant literature on the re-professionalization of the medical profession.

Various managerial types of activities that are enshrined in the competencies represented in the 'Society' domain, have expeditiously become part of most physicians' daily activities. These also include expectations relating to physicians' active involvement in healthcare quality, safety, innovation and sustainability $[4,89,90]$. Unsurprisingly, the increased hybridity in the subsequent complexity of physician's work, allegedly cannibalizing on pure clinical work, patient-physician time, as well as physician's well-being, is often disputed and met with reluctance [91, 92].

5-points Likert scores on agreement:

$1=$ not at all; $2=$ not; $3=$ =medium; $4=$ much; $5=$ very much

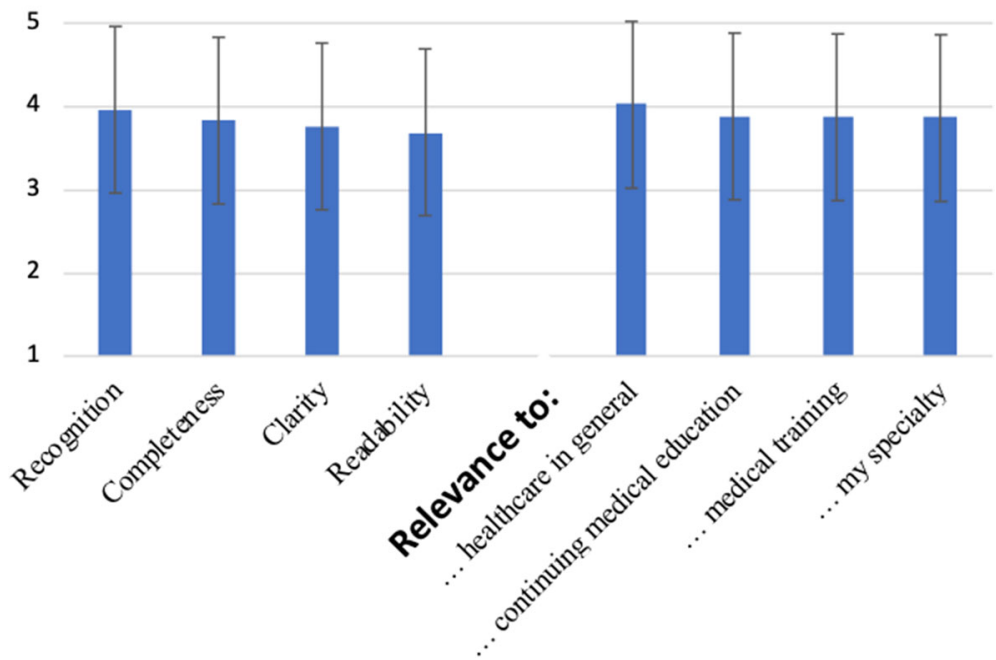

Fig. 5 Face validity scores (mean and SD) of the $12 \mathrm{ML}$ domains of the DML framework 0.2 ( $n=82$ responders) 


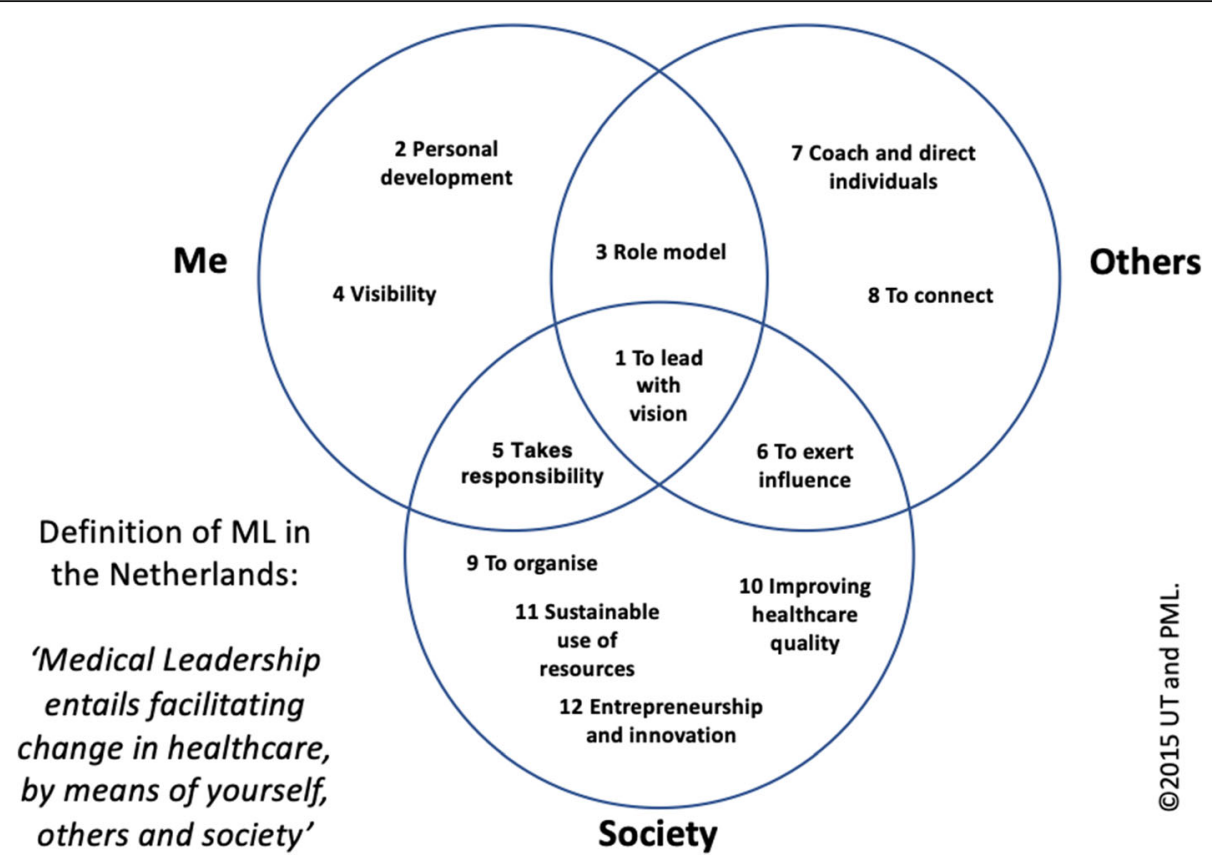

Fig. 6 DML Framework v1.0: Dimensions, competency domains and definition

The framework's dimension 'Others' embodies the paradigmatic shift in physicians' professional positions. Enhanced by a significant influx of information and communication technologies, and by the growing urgency to function within complex, collaborative networks that span pre-existing professional and other boundaries, physicians' interprofessional competences are more relevant than ever before [93]. Hence, physicians are increasingly being seen as agents of change: beyond healthcare's historical professional silos. 'Going beyond the silos' is often referred to as vital in resolving wicked problems that arise from disruptive effects of, for example: system reform; integrated care; e-health; artificial intelligence; and robotics [25, 30, 94-96].

The 'Self' dimension in the framework reflects a rising awareness within the medical community of the significance of physicians' professional self-reflectivity and personal development [91, 92, 97, 98]. The focus on 'soft' skills is relatively new to the medical profession, which is historically educated in more factualknowledge oriented medical sciences. Conceivably, this type of skills might prove beneficial for physicians' effectiveness by living up to their professional roles in dealing with the complexities in their daily activities.

\section{Practical implications}

For ML discourse and practice

As in other countries, the discourses on integrating physicians' new roles are envisioned to strengthen the twenty-first Century Dutch healthcare workforce [89]. However, a transformation of existing tacit knowledge into explicit knowledge is needed to allow effective dissemination [24] of the new roles and accompanying norms, values and behavior as well as subsequent novel interprofessional arrangements that accompany healthcare's institutional change. Our findings indicate that not everyone in the Dutch medical profession is rethinking their professional identity. The DML framework holds the promise of a generation of highly collaborative, flexible, patient-centered, complex-systemready and continuous-improvement-oriented physicians. Some argue this to be a renewal of physicians' decaying social contract with society, or reclamation of their historic authoritarian position [18, 99]. However, strong indications are found of the rise of a twenty-first century physician who is a medical 'boundary spanner' skilled in: (leading) co-creative, interprofessional collaboration; continuous improvement of quality; affordability; and personal development [92]. These medically trained 'agents of change' might actually help solve 'wicked problems' or 'grand challenges' that represent the unprecedented challenges accompanying healthcare transformation [93]. Such a more servant type of leadership, a new 'golden standard' incorporated in physicians' role $[4,16,100]$, concurs with the idea that physicians are also able to take the 'back seat' and enact effective followership [101].

Besides the framework's applicability to institutional or (inter-)professional discussions, the DML framework seems to be ready for use in daily practice [18]. 
Also, a recent interview-based evaluation ${ }^{8}$ revealed its use, varying from structural embedment in a Dutch family medicine residency program, to use during $\mathrm{ML}$ training courses, specialist conferences workshops and reflective-practice sessions by medical specialist groups, as well as its application by individual physicians (e.g., for personal development, or for their mentees/students).

\section{For medical education}

At best, for now, the Dutch ML framework provides a contextualized (i.e., national) 'leadership lens' for educationalists in refinements of redesigns of curricula, as well as to others offering various Dutch ML training programs, that have been burgeoning in the last decade [102]. In its current version, this generic set of ML competencies, which are closely related to safe and effective services in healthcare, might represent a kind of initial 'cognitive foundation' of ML competency development in the Netherlands. As such, it provides one of several stepping-stones for further elaboration of realizing contemporary Dutch physician's effective ML behavior and enactment $[23,103]$.

Concurring with others, we suggest that ML competency development might be importantly harbored within the realms of medical socialization processes [104]. Although these are much debated and dynamic fields of expertise, the arrival of a DML framework might be instrumental, for example, in designing (feedback) instruments for (e.g., behavioral) reflective practice on leadership, complementing more cognitive typed pedagogics $[19,105,106]$. Regardless, we are still far from in-depth know-how relating ML and its educational principles, for example, physicians' 'entrustable leadership activities' and associated behaviors (varying from patient-related, organizational, to political activities) $[22,25,107,108]$.

\section{For ML framework development - a transferable route map?}

Not much comparison data on how to compose a ML framework was available at the onset of designing our study. Our approach contrasts with more top-down, centrally coordinated national ML designs and implementations in other countries [5, 20, 102]. Rather than following a more political process of assembling various stakeholder groups and organizing national sessions, we chose a community of practice approach in which a dedicated research group analyzed various resources,

\footnotetext{
${ }^{8}$ Unpublished findings from 21 exploratory interviews with Dutch physicians (2017), 2 years post-launch of the DML framework version 1.0 .
}

including data from interviews and FG sessions with representatives of relevant stakeholders $[4,22,23,30]$.

To enhance realistic reflections of opinions and behaviors of healthcare's daily practices, critical and equally motivated practitioners from a CoP (PML) were mustered to join the research group ([32, 109]: p. 327). Their independent work, without financial support, we contend, contributed to the group's high degree of autonomy. The long-term commitment of this large group of volunteering practitioners and topic experts was crucial for our goal to avert reproduction of conventional practices. It enabled us to execute a fully independent research group, instead of a 'policy community'. The entire design journey lasted approximately 2.5 years, a period that was characterized by abundant ML related 'knowledge interaction' in the Netherlands, also providing a fruitful 'gestational' phase for the maturing of $M L$ in the field vis-à-vis the actual development of a competency set we named the DML framework version $1.0[35,110]$. Within and beyond this timeframe, the two more entrenched institutional consortium partners, PML and KNMG, prepared for the framework's 'welcome landing', which contributed to the current appetite for ML across the Netherlands [18]. As a result, we think the approach described here was helpful in circumventing long and winding decision-making processes by having representatives of established institutions and authorities within the healthcare system $[5,30]$.

However, the question remains whether our approach has been more effective than alternative approaches elsewhere. A 'short cut' alternative to our approach could have been translating an existing framework, such as MLCF or LEADS $[3,19]$. This has been done with the latter: the originally Canadian LEADS framework was introduced in New Zealand and Australia [3,5]. A detailed comparison between various approaches would require further research.

It is conceivable that other approaches, such as more top-down or 'political' types, can be more effective or less demanding. Secondly, access to national typed published sources on ML might vary. In our case, most of the data that was actually used (in terms of coded fragments) did not come from published materials. This brings about our third consideration: cultural differences [111, 112]. Payment structures; (interprofessional) power distances; relational identities; physicians' economic position; national culture and other differences might affect the creation of a national ML framework [91, 111-114]. Ultimately, those embarking for developing a national ML framework might wisely contemplate such possible factors and consider designing a tailored, hybrid approach, optimally fitting their context.

\section{Strengths and limitations}

First, multiple sources were used for the literature review (snowball searches; topic expert consultation). 
Despite collecting a rich set of data, the uncharted character of ML was reflected in the absence of explicit definitions of the concept or related competencies in the Dutch literature. Our efforts to create a contemporary national taxonomy of a widely acknowledged (but still emerging, hence immature) concept might somehow have impeded our literature searches: through the absence of widely used and homogeneous terminology as well as a relative lack of publications eligible for analysis. Regarding the quality perspective of included studies in our literature search: ML's newness might have resulted, not surprisingly, in the inclusion of primarily qualitative studies which could not offer any empirical facts yet on the content of ML (Table 3). Overall, the literature review contributed only to some extent to our work, while the majority of data used to construct the framework came from interviews and FG sessions.

Furthermore, our use of relatively new phrases in the empirical research might have impacted respondents' feedback. Interpretations of ML's meanings tend to vary from person to person. Yet, the fact that neither comparison with other national frameworks nor feedback during FGs and in surveys provided additional elements of ML, corroborates the comprehensiveness of the framework that resulted from the literature review and interviews. Despite the high time-investments in the interviews and surveys, the respondents' participation was entirely voluntary and non-remunerated. Their relatively high degree of willingness to participate is based on a more-than-average interest in the potential of ML, many being PML associates [109]. Notwithstanding physicians' notorious busy and unpredictable work schedules, often resulting in last minute cancellations, no-shows and non-responses, involving larger samples in future studies may benefit a better understanding of physicians' leadership repertoires.

When reflecting on the survey used for face validity testing, it is relevant to note that perceived 'recognition', 'completeness' and 'relevance' of the 0.2 DML framework was high (Fig. 5). Some of the responses, however, initiated a substantial shortening of version 0.2 , resulting in the final 1.0 version. In our opinion, further work on the framework's validity, could be beneficial. Additional recommended validity-testing approaches include Delphi techniques, for example within various medical specialists' fields [115-117].

\section{Future work}

Various questions are burgeoning due to the relative infancy of ML, possibly guiding further scholarly questions like: How is effective ML best learned and trained? To what extent is effective $M L$ related to personal traits, clinical settings, and medical specialties? How should the 'gap' between knowing-when and actually-doing be bridged? Who should teach ML, and when?

Similar to other novelties or new approaches, the medical profession is more likely to accept changes if based on thoroughly grown evidence. In particular since a ML framework can instill critical reflecting on individual behaviors, it is vital that such frameworks and resulting instruments or tactics meet with highest professional standards. Providing a first generic set of ML competencies, the DML framework 1.0, we think, could impart further endeavoring integration of ML in daily practice as well as education. However, we acknowledge that much more work must be done to enable practical and effective application. Although our work might add to a variety of approaches in designing a national ML framework, more work could help understand which approach under what conditions is most appropriate in a country. Additionally, concurring with previous calls for further research on ML and competency frameworks [118], and reflecting on own research, we propose the following ideas for future research.

Notably, firstly, our framework could use further extensions, such as: 'examples of learning and development opportunities', and vignettes depicting 'examples in practice', such as in early versions of the MLCF in the United Kingdom [19]. Similarly, distinctions between undergraduate, postgraduate and continuing practice could be anticipated, which could instill interesting debate on expectations about ML at physician's various career levels. Desirably, future development of (sufficiently validated) instruments to adequately reflect on actual (micro-)behaviors are welcomed $[23,27,106,119,120]$. Prior work suggests that this is feasible [19, 78, 121]. Such advances might help to evolve ML beyond alleged arid and generic "long [wish]lists of specific competences" ([22, 23, 107]: p.543]).

Relatedly, secondly, effective incorporation of ML in medical education would require more detailed knowledge on what is relevant ('construct-relevant signal'), and what is not ('construct-irrelevant noise'), particularly when measuring or assessing individual ML competencies ([23], p: 54). In the educational perspective, one must take into account: various contextual clinical settings and specialties; physicians' various (clinical, managerial and other) roles; career phases; and variances in their traits and personal interests [23, 121, 122]. Following the statement "the person you are, the leader you are" ([3]: p.4), we note the importance to consider personal traits, demands and preferences when deliberating about ML competency assessments and development. Also, additional efforts to contextualize and personalize ML education might add to current frameworks becoming 'livelier', hence more appealing to physicians, whilst helping to bridge the current void in discipline-specific ML learning [117, 123]. 
Thirdly, we advocate more scholarly work on ML's embedment in the dynamics of medical socialization, self-conceptualization, identity creation and mimicry of personas across physicians' life-long phases of learning [124]. Enculturation of physicians relates to the often debated 'hidden curriculum', renowned for significantly contributing to medical professionalization. This might be one of the suiting pedagogic domiciles for ML development [104]. However, to date, medical enculturation has remained relatively understudied, despite various attempts to integrate ML in curricula and training [125]. The same holds for the effects of (leadership) personas and role models in professional identity development [126]. Thus, more theorizing on and understanding of the role of medical (re) professionalization in healthcare transformation could benefit from design types of research [32], ex-post evaluation implementation and practical use of effective ML related interventions [127], as well as from engaging ethnographically inclined researchers. Such studies might also provide more insights into answering this Catch-22 question: How should $M L$ be taught in the absence of a generation of trainers and mentors adequately educated and trained in $M L$ ?

\section{Conclusion}

The case study presented in this paper intends to provide an accessible reference for others endeavoring a similar canonical knowledge artefact comprising a national vocabulary on ML as a "focal point for a critical discussion" ([24], p., 68) within as well as beyond the medical community in their country [128]. With adequate adaptations, and considering national differences and local aspects, elements of the approaches we have described might be helpful in guiding such efforts [129]. To the best of our knowledge, this paper is the first detailed account of designing a national framework of leadership competencies for physicians, in particularly using a dedicated community of practice ([130]: p. 310).

As to how ML will evolve in the Netherlands and in other nations, relies on various factors [32, 109]. The high degree of similarities between leadership competency frameworks of various healthcare professions suggest that collective co-leadership among all healthcare professionals is on the rise [30]. Future research, in as well as outside of medicine and medical education, is required to better understand consequences of the coming of age of medical and other types of leadership, and how this can benefit the sustaining of quality and affordability of healthcare's complex interprofessional practices [15].

\section{Abbreviations}

CBE: Competency based education; DML: Dutch medical leadership; FG: Focus group; KNMG: Royal Dutch Medical Association; ML: Medical leadership; MLCF: Medical leadership competency framework; PBL: Problembased learning; PML: Platform medical leadership; UT: University of Twente

\section{Acknowledgements}

We thank Marlies de Rond, Suzanne Schoenmaker, Aniek ter Mors, Peter van den Berg, Max Poorthuis, Jamiu Busari and the 2016 Honours 'Processes of Change' class for their participation and dedicated support. We thank the Editors and our Reviewers, Fedde Scheele and Gerald Wickham, for their constructive and valuable criticism on an earlier version of our manuscript which has been instrumental in the process of writing this paper.

\section{Authors' contributions}

WAK constructed the initial design and guided the overall methodological integrity and quality management, with contributions from CPMW. All the authors were involved in further conceptions and design, as well as in data acquisition and analysis, as described in the Methods section. All the authors interpreted the data together. WAK wrote an initial draft of this paper after which all the authors contributed to the various drafts, including the final manuscript. All authors read and approved the final manuscript.

\section{Funding}

None of the authors received remunerations for their input, advice or contributions.

\section{Availability of data and materials}

Data used and analyzed during this study are available online (see: https:// osf.io/qknds/, https://osf.io/b2yeh/, https://osf.io/wdjax/, https://osf.io/kh2vx/, https://osf.io/r8ucj/ and https://osf.io/m93yq/) or available from the corresponding author on reasonable request.

\section{Ethics approval and consent to participate}

Ethical approval was not applicable according to the regulations at the University of Twente under which this study was deployed at the time of interviews and focus groups. This study did not involve any experiment and did not require use of sensitive information relating to an individual. Nevertheless, this study's participants in the interviews, focus groups and surveys consented to have their responses (anonymously) recorded, transcribed and stored. All the data, including interview transcriptions, were kept anonymously.

\section{Consent for publication}

Not applicable.

\section{Competing interests}

The authors declare that they have no competing interests.

\section{Author details}

${ }^{1}$ Faculty of Behavioural, Management and Social Sciences (BMS) Change, Management and Organizational Behavior (CMOB), University Twente, Enschede, The Netherlands. 'DIRMI Foundation, Utrecht, The Netherlands. ${ }^{3}$ University Medical Centre Utrecht, Utrecht Heidelberglaan 100, 3584 CX Utrecht, The Netherlands. ${ }^{4}$ Julius Centre for Health Sciences and Primary Care, University Medical Centre Utrecht, Utrecht Heidelberglaan 100, 3584 CX Utrecht, The Netherlands. ${ }^{5}$ Erasmus Medical Center, Wytemaweg 80, 3015 CP Rotterdam, The Netherlands. ${ }^{6}$ Department of Orthopaedic Surgery, University Medical Center Utrecht, Utrecht Heidelberglaan 100, 3584 CX Utrecht, The Netherlands. ${ }^{7}$ University of Twente, Drienerlolaan 5, Enschede, The

Netherlands. ${ }^{8}$ Institute of Health and Society, University of Oslo, Oslo, Norway. ${ }^{9}$ Department of Continuing Education, University of Oxford, Oxford OX1 2JD, UK

Received: 11 January 2019 Accepted: 9 September 2019 Published online: 28 November 2019

\section{References}

* Records included in the literature review

1. Keijser WA, Poorthuis MB, Tweedie J, Wilderom CPM. Review of determinants of national medical leadership development. BMJ Leader. 2017;1:36-43.

2. Reinertsen $J$, Gosfield AG, Rupp W, Whittington JW. Engaging physicians in a shared quality agenda. IHI innovation series white paper. Cambridge: Institute for Healthcare Improvement; 2007. 
3. Health Workforce Australia. Health LEADS Australia: the Australian health leadership framework. 2013. https://www.aims.org.au/documents/item/352. Accessed 2 Jan 2019.

4. Martin GP, Learmonth M. A critical account of the rise and spread of 'leadership': the case of UK healthcare. Soc Sci Med. 2012;74:281-8.

5. Shannon EA. Health LEADS Australia: implementation and integration into theory and practice. Asia Pac J Health Manag. 2015;10:56-62.

6. McGivern G, Currie G, Ferlie E, Fitzgerald L, Waring J. Hybrid managerprofessionals' identity work: the maintenance and hybridization of medical professionalism in managerial contexts. Publ Admin. 2015;93:412-32.

7. Voogt JJ, Rensen van E, Noordegraaf M, Schneider M. Medisch leiderschap ontrafeld. Ned Tijdschr Geneeskd. 2015;159:A9123 (Dutch).

8. Birden H, Glass N, Wilson I, Harrison M, Usherwood T, Nass D. Teaching professionalism in medical education: a best evidence medical education (BEME) systematic review. BEME guide no. 25. Med Teach. 2013;35:1252-66.

9. Nicolini D. Stretching out and expanding work practices in time and space: the case of telemedicine. Hum Relat. 2007;60:899-920.

10. Nicolini D. Practice theory, work and organization: an introduction. Oxford: Oxford University Press; 2012.

11. Lee TH. Turning doctors into leaders. Harv Bus Rev. 2010;88(4):50-8.

12. Stoller JK. Help wanted: developing clinician leaders. Perspect Med Educ. 2014;3:233-7.

13. West M, Armit K, Loewenthal L, Eckert R, West T, Lee A. Leadership and leadership development in healthcare: the evidence base. London: The Kings Fund; 2015. https://www.kingsfund.org.uk/sites/default/files/field/field_ publication_file/leadership-leadership-development-health-care-feb-2015.pdf Accessed 02 Jan 2019

14. Swensen S, Kabcenell A, Shanafelt T. Physician-organization collaboration reduces physician burnout and promotes engagement: the Mayo Clinic experience. J Healthc Manag. 2016;61:105-27.

15. Lees P, Armit K. Medical leadership: an evidence-free zone? BMJ Leader. 2018;2:52-3.

16. Dath D, Chan M-K, Abbott C. CanMEDS 2015: from manager to leader. Ottawa: The Royal College of Physicians and Surgeons of Canada; 2015.

17. Barry E, Grunberg N, Kleber H. Approaches for curriculum and assessment in leader and leadership education and development programs in American medical schools. Med Ed Publish. 2018;7:23.

18. Berghout MA, Oldenhof L, Fabbricotti IN, Hilders CGJM. Discursively framing physicians as leaders: institutional work to reconfigure medical professionalism. Soc Sci. 2018;212:68-75.

19. NHS Leadership Academy. Medical leadership competency framework: selfassessment. 2012. https://www.leadershipacademy.nhs.uk/wp-content/ uploads/2012/11/NHSLeadership-Framework-MLCFSelfAssessmentTool.pdf. Accessed 2 Jan 2019

20. Sebastian A, Fulop L, Dadich A, et al. Health LEADS Australia and implications for medical leadership. Leadersh Health Serv. 2014:27:355-70.

21. Frank JR, Mungroo R, Ahmad Y, Wang MM, De Rossi S, Horsley T. Toward a definition of competency-based education in medicine: a systematic review of definitions. Med Teach. 2010;32:631-7.

22. ten Cate O, Snell L, Carraccio C. Medical competence: the interplay between individual ability and the health care environment. Med Teach. 2010;32:669-75.

23. Lurie SJ. History and practice of competency-based assessment. Med Educ 2012;46:49-57

24. Abidi SSR. Healthcare knowledge sharing: purpose, practices, and prospects. In: Bali RK, Dwivedi AN. Healthcare knowledge management. Health informatics. New York: Springer; 2007.

25. Thistlethwaite JE, Forman D, Matthews LR, Rogers GD, Steketee C, Yassine T. Competencies and frameworks in interprofessional education: a comparative analysis. Acad Med. 2014;89:869-75.

26. Webb AM, Tsipis NE, McClellan TR, et al. A first step toward understanding best practices in leadership training in undergraduate medical education: a systematic review. Acad Med. 2014;89:1563-70.

27. Carraccio C, Wolfsthal SD, Englander R, Ferentz K, Martin C. Shifting paradigms: from Flexner to competencies. Acad Med. 2002;77:361-7.

28. Noordegraaf M. Hybrid professionalism and beyond. (New) forms of public professionalism in changing organizational and societal contexts. J Profes Org. 2015;2:187-206

29. Swanwick T, McKimm J. What is clinical leadership ... and why is it important? Clin Teach. 2011;8(1):22-6.

30. Reeves S, Fox A, Hodges BD. The competency movement in the health professions: ensuring consistent standards or reproducing conventional domains of practice? Adv Health Sci Educ. 2009;14:451.
31. Greenwood R, Hinings CR, Suddaby R. Theorizing change: the role of professional associations in the transformation of institutionalized fields. Acad Manag J. 2002;45:58-80.

32. McKenney S, Reeves TC. Conducting educational design research. London: Routledge; 2013.

33. Wenger E, McDermott RA, Snyder W. Cultivating communities of practice: a guide to managing knowledge. Boston: Harvard Business School Press; 2002.

34. Sousa VD, Rojjanasrirat W. Translation, adaptation and validation of instruments or scales for use in cross-cultural health care research: a clear and user-friendly guideline. J Eval Clin Pract. 2011;17:268-74.

35. Davies H, Nutley S, Walter I. Why 'knowledge transfer' is misconceived for applied social research. J Health Serv Res Policy. 2008;13(3):188-90.

36. Barnett-Page $E$, Thomas J. Methods for the synthesis of qualitative research: a critical review. BMC Med Res Methodol. 2009;9:1

37. Tong A, Flemming K, Mclnnes E, Oliver S, Craig J. Enhancing transparency in reporting the synthesis of qualitative research: ENTREQ. BMC Med Res Methodol. 2012:12:181.

38. Tong A, Sainsbury P, Craig J. Consolidated criteria for reporting qualitative research (COREQ): a 32-item checklist for interviews and focus groups. Int J Qual Health Care. 2007;19:349-57.

39. Thomas J, Harden A. Methods for the thematic synthesis of qualitative research in systematic reviews. BMC Med Res Methodol. 2008;8:45.

40. Farmer T, Robinson K, Elliott SJ, Eyles J. Developing and implementing a triangulation protocol for qualitative health research. Qual Health Res. 2006;16:377.

41. Babbie E. The practice of social research. 13th ed. Belmont: Wadsworth Publishing; 2011.

42. Spall S. Peer debriefing in qualitative research: emerging operational models. Qual Inq. 1998:4:280-92.

43. Dooley D. Social research methods. 4th ed. Harlow: Pearson Education Limited; 2009.

44. Miles MB, Huberman M, Saldaña J. Qualitative data analysis. A methods sourcebook. 3rd ed. Thousand Oaks: Sage; 2014.

45. Higgins JPT, Green S (editors). Cochrane handbook for systematic reviews of interventions version 5.1.0. The Cochrane Collaboration, 2011. Available from www.handbook.cochrane.org. Accessed 02 Jan 2019.

46. Naumann F. How to develop a search strategy for a Cochrane review. Cochrane Haematological Malignancies Group; 2007. p. 1-10. http:// docplayer.net/11475-How-to-develop-a-search-strategy-for-a-cochranereview.html. Accessed 02 Jan 2019

47. " van Daele B, Haustermans K. Ethical values in managing a department. Radiother Oncol. 2012;103(Suppl 1):S37-8.

48. ${ }^{*}$ Fleuren MAH, Wiefferink $\mathrm{CH}$, Paulussen TGW. Determinants of innovation within health care organizations: literature review and Delphi-study. Int Qual Health Care. 2004;16:107-23.

49. *Bloemen-Vrencken JH, De Witte LP, Engels JP, van den Heuvel WJ, Post MW. Transmural care in the rehabilitation sector: implementation experiences with a transmural care model for people with spinal cord injury. Int J Integr Care. 2005;5:e02.

50. * Scholten RM, van der Grinten TED. The integration of medical specialists in hospitals. Dutch hospitals and medical specialists on the road to joint regulation. Health Policy. 2005;72:165-73.

51. " Prince KJ, van Eijs PW, Boshuizen HP, van der Vleuten CP, Scherpbier AJ. General competencies of problem-based learning (PBL) and non-PBL graduates. Med Educ. 2005;39:394-401.

52. *Van Raak A, Paulus A, Cuijpers R, te Velde C. Problems of integrated palliative care: a Dutch case study of routines and cooperation in the region of Arnhem. Health Place. 2008;14:768-78.

53. * Dückers MLA, Stegeman I, Spreeuwenberg P, Wagner C, Sanders K, Groenewegen PP. Consensus on the leadership of hospital CEO's and its impact on the participation of physicians in improvement projects. Health Policy. 2009;91:306-13.

54. ${ }^{*}$ Klopper-Kes AHJ, Meerdink N, van Harten WH, Wilderom CMP. Stereotypical images between physicians and managers in a hospitals. J Health Organ Manag. 2009;23:216-24.

55. "Berkenbosch L, Bax M, Scherpbier A, Heyligers I, Muijtjens AMM, Busari JO. How Dutch medical specialists perceive the competencies and training needs of medical residents in healthcare management. Med Teach. 2013;35:10901102.

56. " Cramm JM, Strating MMH, Nieboer AP. Development and validation of a short version of the partnership self-assessment tool (PSAT) among professionals in Dutch disease-management partnerships. BMC Res Notes. $2011 ; 4: 224$. 
57. * Klopper-Kes AHJ, Meerdink N, Wilderom CPM, van Harten WH. Effective cooperation influencing performance: a study in Dutch hospitals. Int J Qual Health Care. 2011;23:94-9.

58. " Schreuder JAH, Roelen CAM, van Zweeden NF, Jongsma D, van der Klink $J \mathrm{~J}$, Groothoff JW. Leadership styles of nurse managers and registered sickness absence among their nursing staff. Health Care Manag Rev. 2011; 36:58-66.

59. * Teunissen PW, Westerman M. Junior doctors caught in the clash: the transition from working to learning explored. Med Educ. 2011;45:968-70.

60. * van der Lee N, Westerman M, Fokkema JP, Van Der Vleuten CP, Scherpbier AJ, Scheele F. The curriculum for the doctor of the future: messages from the clinician's perspective. Med Teach. 2011;33:555-61.

61. * Berben SAA, Meijs THJM, Van Grunsven PM, Schoonhoven L, van Achterberg T. Facilitators and barriers in pain management for trauma patients in the chain of emergency care. Injury. 2012;43:1397-402.

62. *Buljac-Samardžic M, van Woerkom M, Paauwe J. Team safety and innovation by learning from errors in long-term care settings. Health Care Manag Rev. 2012;37:280-91.

63. ${ }^{*} \varnothing v$ retveit J, Klazinga N. Learning from large-scale quality improvement through comparisons. Int J Qual Health Care. 2012;24:463-9.

64. ${ }^{*}$ Smith PC, Anell A, Busse R, Crivelli L, Healy J, Karin A, Lindahl F, Westert G, Kene T. Leadership and governance in seven developed health systems. Health Policy. 2012;106:37-49.

65. ${ }^{*}$ Aij KH, Simons FE, Widdershoven GAM, Visse M. Experiences of leaders in the implementation of lean in a teaching hospital_-barriers and facilitators in clinical practices: a qualitative study. BMJ Open. 2013;3:003605.

66. * Berkenbosch L, Brouns JW, Heyligers I, Busari JO. How Dutch medical residents perceive their competency as manager in the revised postgraduate medical curriculum. Postgrad Med J. 2011;87:680-7.

67. ${ }^{*}$ Cramm JM, Phaff S, Nieboer AP. The role of partnership functioning and synergy in achieving sustainability of innovative programmes in community care. Health Soc Care Comm. 2013:21:209-15.

68. * Cramm JM, Strating MMH, Nieboer AP. The influence of organizational characteristics on employee solidarity in the long-term care sector. J Adv Nurs. 2013;69:526-34.

69. "Elshout R, Scherp E, van der Feltz-Cornelis CM. Understanding the link between leadership style, employee satisfaction, and absenteeism: a mixed methods design study in a mental health care institution. Neuropsych Dis Treatm. 2013;9:823-37

70. ${ }^{*}$ Huis A, Holleman G, Van Achterberg T, Grol R, Schoonhoven L, Hulscher M. Explaining the effects of two different strategies for promoting hand hygiene in hospital nurses: a process evaluation alongside a cluster randomised controlled trial. Implement Sci. 2013;8:41.

71. * Ijkema R, Langelaan M, van de Steeg L, Wagner C. What impedes and what facilitates a quality improvement project for older hospitalized patients? Int J Qual Health Care. 2014;26:41-8.

72. "Witman Y, van den PCM K, DDM B. Medische professionals over hun kernwaarden. Het belang van praktijkverhalen en intrinsieke motivatie. Ned Tijdschr Geneeskd. 2013;157:A5698 (Dutch).

73. Wolfswinkel J, Furtmueller E, Wilderom C. Using grounded theory as a method for rigorously reviewing literature. Eur J Inf Syst. 2013;22:45-55.

74. JBI. The Joana Briggs Institute. Joanna Briggs Institute Levels of Evidence and Grades of Recommendation Working Party. http://joannabriggs.org/jbiapproach.html\#tabbed-nav=Levels-of-Evidence. Accessed 02 Jan 2019.

75. Armola RR, Bourgault AM, Halm MA, et al. AACN levels of evidence: What's new? Crit Care Nurse. 2009;29:70-3.

76. Creswell JW. Research design qualitative and quantitative approaches. 3rd ed. California: Sage; 1994

77. Academy of Medical Royal Colleges and NHS Institute. Medical Leadership Competency Framework. 3rd ed; 2010. https://www.leadershipacademy.nhs.uk/ wp-content/uploads/2012/11/NHSLeadership-Leadership-Framework-MedicalLeadership-Competency-Framework-3rd-ed.pdf . Accessed 02 Jan 2019

78. FMLM. Faculty of Medical Leadership and Management. Developing medical leadership. A toolkit for doctors in postgraduate training. 2018. Available at: https://www.fmlm.ac.uk/sites/default/files/content/ resources/attachments/Developing medical leadership toolkit.pdf. Accessed 23 Aug 2018).

79. Clark J, Armit K. Leadership competency for doctors: a framework. Leadersh Health Serv. 2010;23:115-29.

80. Gerring J. Social science methodology: a unified framework. 6th ed. Cambridge: Cambridge University Press; 2012.
81. Mason M. Sample size and saturation in PhD studies using qualitative interviews. Forum: Qual Health Res. 2010;11:3.

82. Guest G, Bunce A, Johnson L. How many interviews are enough? An experiment with data saturation and variability. Field Methods. 2006;18:59-82.

83. Glaser BG, Strauss AL. The discovery of grounded theory: strategies for qualitative research. New York: Aldine De Gruyter; 1967.

84. Moran M, Steketee C, Marles K. Health LEADS Australia and Interprofessional education. In: Dawn F, editor. Leadership and collaboration further developments for interprofessional education. New York: Palgrave Macmillan; 2015. p. 182-98.

85. Giannarou L, Zervas E. Using Delphi technique to build consensus in practice. Int J Bus Sci Appl Manag. 2014;9:65-82.

86. Seale C. The quality of qualitative research. Thousand Oaks: Sage; 1999.

87. Beaton DE, Bombardier C, Guillemin F, Ferraz MB. Guidelines for the process of cross-cultural adaptation of self-report measures. Spine. 2000;25:3186-91.

88. Corbin JM, Strauss A. Grounded theory research: procedures, canons, and evaluative criteria. Qual Sociol. 1990;13:3.

89. Reay $T$, Goodrick E, Hinings B. Institutionalization and professionalization. In: Ferlie E, Montgomery K, Reff Pedersen A, editors. The Oxford handbook of health care management. New York: Oxford University Press; 2016.

90. Denis $J$, van Gestel N. Medical doctors in healthcare leadership: theoretical and practical challenges. BMC Health Serv Res. 2016;16:158.

91. Ratcharak P, Spyridonidis D, Vogel B. Emotional dynamics at the relational level of self: the case of healthcare hybrid professionals. In: Petitta L, Härtel CEJ, Ashkanasy NM, Wilfred JZ, editors. Individual, relational, and contextual dynamics of emotions. Research on emotion in organizations, vol. 14. Bingley: Emerald Publishing Limited; 2018. p. 71-94.

92. Ewert B. Focusing on quality care rather than "checking boxes": how to exit the labyrinth of multiple accountabilities in hybrid healthcare arrangements. Publ Admin. 2018. https://doi.org/10.1111/padm.12556.

93. Chreim S, Langley A, Comeau-ValleÂe M, Huq J, Reay T. Leadership as boundary work in healthcare teams. Leadership. 2013:9:201-28.

94. Rogers GD, Thistlethwaite JE, Anderson ES, Dahlgren MA, Grymonpre RE, Moran M, Samarasekera DD. International consensus statement on the assessment of interprofessional learning outcomes. Med Teach. 2017:39:347-59.

95. Heaphy E, Gittel JH, Leana C, Sluss D, Ballinger G. The changing nature of work relationships. Ac Manag R. 2016;41:184-6.

96. Loh E. Medicine and the rise of the robots: a qualitative review of recent advances of artificial intelligence in health. BMJ Leader. 2018;2:59-63.

97. Hilton SR, Slotnick HB. Protoprofessionalism: how professionalisation occurs across the continuum of medical education. Med Educ. 2010;39:58-65.

98. Aukes LC, Geertsma J, Cohen-Schotanus J, Zwierstra RP, Slaets JP. The development of a scale to measure personal reflection in medical practice and education. Med Teach. 2007;29:177-82.

99. Birden H, Glass N, Wilson I, Harrison M, Usherwood T, Nass D. Defining professionalism in medical education: a systematic review. Med Teach. 2014; 36:47-61.

100. Lo D, McKimm J, Till A. Transformational leadership: is this still relevant to clinical leaders? Brit J Hosp Med. 2018;79:344-7.

101. Reay T, Goodrick E, Casebeer A. Getting leopards to change their spots: co-creating a new professional role identity. Ac Manag J. 2017;60:1043-70.

102. McKimm J, O'Sullivan H. Developing and assessing medical leadership. Br J Hosp Med (Lond). 2012;73:484-5.

103. Westera W. Competences in education: a confusion of tongues. J Curric Stud. 2001;33:75-88

104. Miller CJ, Till A, McKimm J. Exploring UK health-care providers' engagement of trainee doctors in leadership. Br J Hosp Med. 2018;79(5):279-83.

105. Wouda JC, van de Wiel HB. The communication competency of medical students, residents and consultants. Patient Educ Couns. 2012;86:57-62.

106. Chesluk BJ, Bernabeo E, Hess B, Lynn LA, Reddy S, Holmboe ES. A new tool to give hospitalists feedback to improve interprofessional teamwork and advance patient care. Health Aff. 2012;31:2485-92.

107. ten Cate O, Scheele F. Competency-based postgraduate training: can we bridge the gap between theory and clinical practice? Acad Med. 2007:82:542-7.

108. Boursicot K, Etheridge L, Setna Z, Sturrock A, Ker J, Smee S, Sambandam E. Performance in assessment: consensus statement and recommendations from the Ottawa conference. Med Teach. 2011;33:370-83.

109. Bereiter C. Design Research for Sustained Innovation. Cogn Stud (Japan). 2002;9:321-7 
110. Cruess RL, Cruess SR, Steinert Y. Medicine as a community of practice: implications for medical education. Acad Med. 2018;93(2):185-91.

111. Hofstede GH. Cultures and organizations: software of the mind. 2nd ed. New York: McGraw-Hill; 1997.

112. Hofstede G. Cultural differences in teaching and learning. Int J Intercult Relat. 1986;10(3):301-20.

113. Jippes M, Majoor GD. Influence of national culture on the adoption of integrated medical curricula. Adv Health Sci Educ. 2011;16:5-16.

114. Davies HTO, Nutley SM, Mannion R. Organisational culture and quality of health care. BMJ Qual Saf. 2000;9:111-9.

115. Sandelowski M, Barroso J. Handbook for synthesizing qualitative research. New York: Springer Publishing Company; 2007.

116. Raghav PR, Kumar D, Bhardwaj P. Experience of Delphi technique in the process of establishing consensus on core competencies. Intern J Appl Basic Med Res. 2016;6:191-4.

117. Turner S, Ming-Ka C, McKimm J, Dickson G. Discipline-specific competencybased curricula for leadership learning in medical specialty training: a critical review of the literature. Leadersh Health Serv. 2018;31:152-66.

118. Montgomery A. Physicians as leaders: are we trying to fit square pegs into round holes? BMJ Leader. 2018;2:128-31.

119. Turnbull J, Cunnington J, Unsal A, Norman G, Ferguson B. Competence and cognitive difficulty in physicians: a follow-up study. Acad Med. 2006;81:915-8

120. Campion M, Fink A, Ruggeberg BJ, Carr L, Phillips GM, Odman RB. Doing competencies well: best practices in competency modeling. Person Psych. 2011;64:225-62

121. Stringfellow TD, Rohrer RM, Loewenthal L, Gorrard-Smith C, Sheriff IHN, Armit K, Lees PD, Spurgeon PC. Defining the structure of undergraduate medical leadership and management teaching and assessment in the UK. Med Teach. 2015;37:747-54.

122. Straus SE, Soobiah C, Levinson W. The impact of leadership training programs on physicians in academic medical centers: a systematic review. Acad Med. 2013;88:710-23.

123. Jaye C, Egan T, Parker S. 'Do as I say, not as I do': medical education and Foucault's normalizing technologies of self. Anthropol Med. 2006;13:141-55.

124. Jackson SE, Joshi A, Erhardt NL. Recent research on team and organizational diversity: SWOT analysis and implications. J Manag. 2003:29:801-30.

125. Gordon J, Markham P, Lipworth W, Kerridge I, Little M. The dual nature of medical enculturation in postgraduate medical training and practice. Med Educ. 2012;46:894-9026.

126. Richardson A, Loubier C. Intersectionality and leadership. Intern J Lead Stud. 2008;:142-61

127. Dixon-Woods M, Bosk CL, Aveling EL, Goeschel CJ, Pronovost PJ. Explaining Michigan: developing an ex post theory of a quality improvement program. Milbank Q. 2011;89:167-205.

128. Stake RE. Qualitative case studies. In: Denzin NK, Lincoln YS, editors. The sage handbook of qualitative research. Thousand Oaks: Sage Publications Ltd; 2005. p. 443-66.

129. Shannon T. Medical Leadership - moving from the dark side towards the age of enlightenment. $201 \mathrm{https}$ //frectal.com/2014/09/30/medicalleadership-moving-from-the-dark-side-towards-the-age-of-enlightenment/. Accessed on 2 Jan 2019

130. Riessman C. A thrice-told tale: reader and audience response to an illness narrative. In: Hurwitz B, Greenhalgh T, Skultans V, editors. Narrative research in health and illness. London: BMJ Publications; 2004. p. 309-24.

\section{Publisher's Note}

Springer Nature remains neutral with regard to jurisdictional claims in published maps and institutional affiliations.

Ready to submit your research? Choose BMC and benefit from:

- fast, convenient online submission

- thorough peer review by experienced researchers in your field

- rapid publication on acceptance

- support for research data, including large and complex data types

- gold Open Access which fosters wider collaboration and increased citations

- maximum visibility for your research: over $100 \mathrm{M}$ website views per year

At BMC, research is always in progress.

Learn more biomedcentral.com/submissions 